\title{
$\alpha$-ketoglutarate augments prolyl hydroxylase-2 mediated inactivation of phosphorylated-Akt to inhibit induced-thrombosis and inflammation
}

\author{
Nishith M Shrimali' ${ }^{1}$, Sakshi Agarwal', Simrandeep Kaur ${ }^{1}$, Sulagna Bhattacharya ${ }^{1}$, \\ Sankar Bhattacharyya ${ }^{2}$, Josef T Prchal ${ }^{3}$ and Prasenjit Guchhait ${ }^{1, *}$
}

\author{
Author affiliations \\ ${ }^{1}$ Regional Centre for Biotechnology; National Capital Region Biotech Science Cluster, Faridabad, \\ India. \\ ${ }^{2}$ Translational Health Science Technology Institute; National Capital Region Biotech Science \\ Cluster, Faridabad, India. \\ ${ }^{3}$ Department of Medicine, University of Utah School of Medicine \& Huntsman Cancer Center and \\ George E. Whalen Veteran's Administration Medical Center, Salt Lake City, UT, USA. \\ ${ }^{*}$ Correspondence to Prasenjit Guchhait at prasenjit@rcb.res.in
}

\begin{abstract}
Phosphorylation of Akt (pAkt) regulates multiple physiological and pathological processes including thrombosis and inflammation. In an approach to inhibit the pathological signalling of pAkt by prolyl-hydroxylase-2 (PHD2) we employed $\alpha$-ketoglutarate $(\alpha K G)$, a cofactor of PHD2. Octyl- $\alpha \mathrm{KG}$ supplementation to platelets promoted PHD2 activity through elevated intracellular $\alpha \mathrm{KG}$ :succinate ratio and reduced aggregation in vitro by suppressing pAkt1(Thr308). Augmented PHD2 activity was confirmed by increased hydroxylated-proline alongside enhanced binding of PHD2 to pAkt in $\alpha \mathrm{KG}$-treated platelets. Contrastingly, inhibitors of PHD2 significantly increased pAkt1 in platelets. Octyl- $\alpha \mathrm{KG}$ followed similar mechanism in monocytes to inhibit cytokine secretion in vitro. Our data also describe a suppressed pAktl and reduced activation of platelet and leukocyte obtained from mice supplemented with dietary- $\alpha \mathrm{KG}$, unaccompanied by alteration in
\end{abstract}


their counts. Dietary- $\alpha \mathrm{KG}$ significantly reduced clot formation and leukocyte accumulation in various organs including lung of mice treated with thrombosis-inducing agent carrageenan. Importantly, we observed a significant rescue effect of dietary- $\alpha \mathrm{KG}$ on inflamed lung of SARSCoV-2 infected hamsters. $\alpha \mathrm{KG}$ significantly reduced leukocyte accumulation, clot formation and viral load alongside downmodulation of pAkt in lung of the infected animals. Therefore, our study suggests a safe implementation of dietary- $\alpha \mathrm{KG}$ in prevention of Akt-driven anomalies including thrombosis and inflammation, highlighting a better pulmonary management in COVID-19.

\section{Introduction}

The serine-threonine kinase Akt, also known as protein kinase B (PKB), contributes to a broad range of cellular functions including cell survival, proliferation, gene expression and migration of cells of most lineages. Akt plays a central role in both physiological and pathological signalling mechanisms. Upon exposure to stimuli, Akt is recruited to the cell membrane by phosphoinositide 3-kinase (PI3K), where it is phosphorylated by membrane associated 3-phosphoinositidedependent kinase-1 (PDK1) and therefore activated. Among the three isoforms, Akt1 is widely expressed in most of the cell types in both human and mice (1-5). The poignant role of PI3K-Akt signalling is well investigated in platelet activation and functions including aggregation, adhesion and thrombus formation (1-7). Platelets from Akt1-/- mice displayed an increased bleeding time and in ex vivo their platelets minimally responded to agonists (3). Studies using inhibitors to Akt including SH-6, triciribine and Akti-X describe the important role of this signaling adaptor molecule in platelets functions including aggregation, clot formation and granule secretion in vitro and in vivo $(4,8-9)$.

Extensive studies have reported the crucial involvement of the PI3K-Akt pathway in the regulation of immune cell function in a broad range of inflammatory diseases such as rheumatoid arthritis, multiple sclerosis, asthma, chronic obstructive pulmonary disease, psoriasis, and atherosclerosis (10-15). Besides, the pathological signalling of Akt is well reported in the progression cancer or tumor cells (16). Activation of PI3K-Akt pathway is highly relevant in infections like SARS-CoV-2 (17-18), SARS-CoV (19), Dengue and Japanese Encephalitis (20) viruses. Akt signalling has been found to be pivotal for the virus entry and replication in host cells. Therefore, extensive reports suggest Akt as a potential therapeutic target in various disease 
conditions (21). The PI3K-Akt inhibitor wortmannin has been used to alleviate the severity of inflammation and improve the survival rate in rats with induced severe acute pancreatitis. Akt1-/mice showed a markedly reduced carrageenan-induced paw edema and related inflammation alongside a significant decrease in neutrophil and monocyte infiltration (22). Studies using inhibitors such as Akti-8 and Akt-siRNA describe the crucial regulatory role of Akt in inflammatory response of monocytes and macrophages in vitro and in vivo (23-24).

In the context of regulation of the Akt pathway, a study has reported that phosphorylated Akt1 (pAkt1) is hydroxylated by an oxygen-dependent enzyme, prolyl hydroxylase 2 (PHD2). The pAkt1 undergoes prolyl hydroxylation at Pro125 and Pro313 by PHD2 in a reaction decarboxylating $\alpha$-ketoglutarate $(\alpha \mathrm{KG})$. This promotes von Hippel-Lindau protein (pVHL) binding to the hydroxylated site. pVHL then interacts with protein phosphatase 2A (PP2A), which dephosphorylates the Thr308 site, resulting in Aktl inactivation (25).

In this study, we describe a heretofore unreported role of PHD2 in the regulation of platelet and monocyte functions by inactivating pAkt1. Supplementation with dietary $\alpha \mathrm{KG}$, a metabolite of TCA cycle and a cofactor of PHD2, appears to be a potent suppressor of pAkt, significantly reducing clot formation and leukocyte accumulation and related thrombotic and inflammatory events in mice treated with thrombosis-inducing agent like carrageenan (26). Further, we show a rescue effect of $\alpha \mathrm{KG}$ on lung inflammation in SARS-CoV-2 infected hamsters. SARS-CoV-2 infection to golden hamsters induces significant inflammation of the bronchial epithelial cells and lung, in turn increases the disease severity between days 3-5 (27). SARS-CoV-2 significantly increases phosphorylation of Akt1(Thr308) (17), a known target of PHD2, in infected cells. We describe that dietary $\alpha \mathrm{KG}$ significantly reduced clot formation, inflammation and viral load in conjunction with downmodulation of pAkt in the lungs of hamsters with SARS-CoV-2 infection.

\section{Results}

Agonist mediated platelet activation is directly related to phosphorylation of Akt1 but inversely with prolyl-hydroxylase activity of PHD2

We checked the presence of all 3 isoforms PHD1, PHD2 and PHD3 in platelets (Figure 1A). A recent report has shown that PHD2 hydroxylates the proline residues of phosphorylated Akt1(Thr308) [pAkt1(Thr308)] eventually leading to inactivation (25). Therefore, we measured phosphorylation status of Akt1 in agonist-activated platelets and check if the PHD2 activity is 
functionally relevant. As expected, the phosphorylation of Pan-Akt or Akt1(Thr308) was increased with higher concentrations of agonists like collagen (Figure 1B-D) and ADP (Supplemental Figure 1A), indicating an insufficient activity of PHD2 enabling the hydroxylation of elevated level of pAkt after agonist stimulation. To confirm the prolyl-hydroxylation of elevated level of pAkt after agonist stimulation. To confirm prolyl-hydroxylation activity of PHD2, we measured expression of other known substrates of the enzyme, such as HIF1 $\alpha$ and HIF2 $\alpha$ in agonist-activated platelets. We observed stabilization of both HIF $1 \alpha$ and HIF2 $\alpha$ in activated platelets under normal oxygen condition or normoxia (Figure 1B). It pertinently raised the speculation that the enzymatic activity of PHD2 remains below the threshold level to inactivate pAkt, which heightens in platelets after agonist stimulation. We then tested whether agonist or other chemical induced alteration in prolylhydroxylase activity of PHD2 can in turn alter the activation status of pAkt in platelet?

\section{Supplementation of octyl $\alpha$ KG inhibits Akt1 phosphorylation by augmenting PHD2 activity}

To alter the enzymatic activity of PHD2, we used $\alpha$-ketoglutarate ( $\alpha \mathrm{KG}$, a cofactor of PHD2) and dimethyl ketoglutarate (DKG, an inhibitor to PHD2). Octyl $\alpha$ KG (a membrane-permeating form) supplementation significantly decreased the collagen-induced phosphorylation of Akt1(Thr308) or pan-Akt, and also destabilized HIF $1 \alpha$ and HIF2 $\alpha$ in platelets under normoxia (Figure 1E-G). Interaction of pAkt1 with PHD2 was confirmed by immunoprecipitation of PHD2 followed by immunoblotting for pAkt1(Thr308). A significantly increased binding of PHD2 to pAkt1(Thr308) in presence of $\alpha \mathrm{KG}$, suggests a pre-existing condition of elevated prolyl-hydroxylation and inactivation of pAkt1 in collagen-activated platelets (Figure 1H). In order to check the enzymatic activity of PHD2 on pAkt, immunoprecipitation was performed using pan-pAkt antibody and immunoblotted for hydroxy proline. An increased level of pAkt-bound hydroxy proline (Figure $1 \mathrm{H})$, suggests an elevated hydroxylation of proline on pAkt in platelets after $\alpha \mathrm{KG}$ supplementation. On the other hand, the PHD2 inhibitor DKG promoted the Akt phosphorylation (Figure 1E-G). Other known inhibitor of PHD2, such as ethyl-3-4-dihydroxybenzoic acid (DHB) also exhibited an elevated Akt phosphorylation (Supplemental Figure 1B). Although, $\alpha \mathrm{KG}$ and DKG altered the enzymatic function of the PHD2 to regulate pAkt, neither of the treatments altered the expression of PHD2 in platelets significantly (Figure 1E). We examined the effect of $\alpha \mathrm{KG}$ on PI3K, activator of Akt. Our data show no significant effect of $\alpha \mathrm{KG}$ on the expression of phosphorylated PI3K(p55) (Supplemental Figure 1C), thus, confirming its specific target on pAkt. 
Octyl $\alpha$ KG supplementation suppresses agonist-induced activation, aggregation and thrombus formation of platelet while PHD2 inhibitors enhance it

Octyl $\alpha \mathrm{KG}$ supplementation suppressed the expression of cell surface activation markers such as P-selectin, phosphatidylserine (PS) and PAC-1 binding to GPIIbIIIa integrin on collagen-activated platelets (Figure 1J-L) and microparticle release from activated platelets (Supplemental Figure 3) in a concentration-dependent manner in vitro. In contrast, DKG enhanced the above parameters (Figure 1J-L). Similarly, $\alpha$ KG suppressed platelet aggregation induced by collagen (Figure 1MN) or ADP (Supplemental Figure 4A-B) in a concentration-dependent manner, but DKG enhanced it. Another PHD2 inhibitor DHB also enhanced collagen-induced platelet aggregation (Supplemental Figure 4C-D). Further, our data show that platelet thrombus formation was increased in a dose-dependent manner when whole blood was treated with DKG and perfused under flow shear condition on immobilized collagen surface. $\alpha$ KG significantly suppressed DKGinduced thrombus formation (Figure 1O-P). Collagen-activated platelets secreted large amount of sphingosine-1-phosphate (S1P), known stimulator of monocytes, which too was reduced by $\alpha \mathrm{KG}$ supplementation (Figure 1Q).

Octyl $\alpha$ KG supplementation significantly suppresses monocyte functions by augmenting PHD2 activity

We then investigated the PHD2-mediated inhibition of pAkt1(Thr308) in monocytes, isolated from healthy individuals and activated with either S1P or LPS after pre-treatment with octyl $\alpha$ KG in vitro. Our data show that the $\alpha \mathrm{KG}$ supplementation decreased pAkt1(Thr308) and increased degradation of HIF2 $\alpha$ (Figure 2A-C). Simultaneous suppression in secretion of inflammatory cytokines including, IL1 $\beta$, IL6, TNF $\alpha$ and IL10 was observed (Figure 2D-G). Similar outcomes were observed in monocytes exposed to LPS after pre-treatment of $\alpha \mathrm{KG}$ (Supplemental Figure 5). We then confirmed that the above mechanism of $\alpha \mathrm{KG}$-induced suppression of monocyte activation is mediated primarily by pAkt, independent of HIF $\alpha$. In HIF1 $\alpha$-depleted U937 monocytic cells (detailed protocol of shRNA-mediated depletion is mentioned in Supplemental Figure 6), $\alpha \mathrm{KG}$ supplementation significantly reduced cytokine secretion (Figure 2H-I) alongside downmodulated pAkt1(Thr308) (Figure 2J-K).

$\alpha$ KG-mediated suppression of pAkt in monocyte is mediated by PHD2 
To confirm that $\alpha \mathrm{KG}$-mediated suppression of pAkt in monocyte is mediated by PHD2, we performed the above experiment in PHD2-depleted U937 monocytic cells. Our data show that S1P-induced elevation of pAkt1 was not significantly suppressed by octyl $\alpha \mathrm{KG}$ in PHD2-depleted cells (Figure 2L-M), highlighting the role of PHD2 in the inactivation of pAkt1.

Elevated intracellular ratio of $\alpha \mathrm{KG}$ to succinate correlates with augmented activity of PHD2 in platelet and monocyte after octyl $\alpha$ KG supplementation

To ascertain the mechanism of augmentation of PHD2 activity we measured an elevated level of intracellular $\alpha \mathrm{KG}$ in collagen-activated platelets after octyl $\alpha \mathrm{KG}$ supplementation, although the level was unaltered in collagen-activated platelets compared to resting platelets in vitro (Figure 3A). Since, succinate, a product of $\alpha$ KG-dependent dioxygenase reaction in TCA cycle, inhibits PHD2 function; we measured its intracellular level and found no significant change in platelets after collagen activation as well as post $\alpha \mathrm{KG}$ supplementation (Figure 3B). However, the intracellular $\alpha \mathrm{KG}$ to succinate ratio was elevated in collagen-activated platelets after $\alpha \mathrm{KG}$ supplementation, which might have played a role in augmentation of PHD2 activity (Figure 3C) as suggested by others (28) as well as our recent work (29). The intracellular level of other metabolites such as fumarate and pyruvate were found unaltered (Supplemental Figure 7A-B). We observed elevated level of lactate in the supernatant of activated platelets, which was reduced by $\alpha \mathrm{KG}$ supplementation (Figure 3D).

We observed a similar elevation of intracellular $\alpha \mathrm{KG}$ to succinate ratio in S1P-stimulated monocytes after octyl $\alpha \mathrm{KG}$ supplementation, although the ratio was unaltered in SIP-activated monocytes compared to untreated monocytes (Figure $3 \mathrm{E}-\mathrm{G}$ ), which might have played a role in augmentation of PHD2 activity.

\section{Supplementation of dietary $\alpha$ KG significantly inhibits platelet aggregation in mice}

We investigated whether the supplementation of dietary $\alpha \mathrm{KG}$ inhibits platelet aggregation in mice, and observed that $1 \% \alpha \mathrm{KG}$ via drinking water for 24 and $48 \mathrm{hrs}$ (experimental detailed is mentioned in Figure 4A) significantly inhibited platelet aggregation ex vivo in response to agonists such as collagen (Figure 4B-C) and ADP (Supplemental Figure 9C). The above $\alpha \mathrm{KG}$ supplementation did not alter the counts of platelets and WBCs in mice (Supplemental Figure 8AC), suggesting a safe implementation of the metabolite. In a recent work, we have mentioned the safe rescue effect of $1 \%$ dietary $\alpha \mathrm{KG}$ in mice exposed to hypoxia treatment (29). 
Elevated intracellular ratio of $\alpha \mathrm{KG}$ to succinate relates augmented activity of PHD2 in platelet and monocyte of mice with dietary $\alpha$ KG supplementation

We have investigated the enzymatic activity of PHD2 in mice from above experiment. Our data show that the dietary $\alpha \mathrm{KG}$ supplementation elevated $\alpha \mathrm{KG}$ level in plasma (Figure 4D) and also in platelets (Figure 4E). An increased intracellular ratio of $\alpha \mathrm{KG}$ to succinate in platelets (Figure 4FG) might have augmented PHD2 activity. In fact, the decreased expression of pAkt1 and HIF2 $\alpha$ in platelets of $\alpha \mathrm{KG}$-supplemented mice, confirmed the augmented-activity of PHD2 (Figure 4HI). The platelets from $\alpha \mathrm{KG}$-supplemented mice showed a reduced secretion of inflammatory mediator S1P (Figure 4J). Similarly, PBMCs collected from $\alpha$ KG-supplemented mice displayed elevated level of intracellular $\alpha \mathrm{KG}$ as well as $\alpha \mathrm{KG}$ to succinate ratio (Figure 4K-M) along with downmodulation of pAkt and HIF $2 \alpha$ (Figure 4N-O). We then tested the effect of dietary $\alpha \mathrm{KG}$ supplementation in animal models of induced thrombosis and inflammation.

Dietary $\alpha$ KG significantly inhibits carrageenan-induced thrombosis and inflammation in mice

Dietary supplementation of $\alpha \mathrm{KG}$ (starting at 24 or $48 \mathrm{hrs}$ before carrageenan treatment) significantly reduced the tail thrombosis (Figure 5B-C) and clot formation in lungs and liver (Figure 5D-G, Supplemental Figure 10A-B), as well as, accumulation of leukocytes in lungs (Figure 5H-I, Supplemental Figure 11) of mice exposed to carrageenan intraperitoneal treatment for $48 \mathrm{hrs}$. $\alpha \mathrm{KG}$ supplementation also suppressed the levels of inflammatory cytokines such as IL1 $\beta$, IL6 and TNF $\alpha$ in plasma of the carrageenan treated mice (Figure 5J-M). Besides, $\alpha \mathrm{KG}$ supplemented mice when treated with carrageenan locally at abdomen and for a short period (3-6 hrs), displayed less accumulation of leukocytes including monocytes and neutrophils, and leukocyte-platelet aggregates, as well as, decreased pro-inflammatory agents such as myeloperoxidase (MPO) in the peritoneum (Figure 5N-T; Supplemental Figure 12B-C).

Dietary $\alpha$ KG significantly inhibits inflammation and thrombosis alongside downmodulation of pAkt in lung of the hamsters infected with SARS-CoV-2

A recent report suggested the increased expression of pAkt1(Thr308) in human alveolar epithelial type 2 cells after SARS-CoV-2 infection (17), we also observed a similar elevated expression of pAkt1(Thr308) in human liver Huh7 cell line infected with this virus. As expected, we observed a significant reduction in pAkt1(Thr308) expression along with decreased IL6 secretion in these 
infected cells after octyl- $\alpha$ KG supplementation (Figure 6A-C), but viral load did not change significantly (Figure 6D). We then tested the rescue effect of dietary $\alpha \mathrm{KG}(1 \%)$, administered via drinking water and oral gavage (protocol of treatment is mentioned in Figure 6E), and observed significantly reduced clot injury spots on lung in SARS-CoV-2 infected hamsters (Figure 6F). The histopathology data showed a significantly reduced intravascular clot formation (Figure 6G-H) and leukocytes accumulation in alveolar spaces (Figure 6I-J) in the lung of SARS-CoV-2 infected hamsters after $\alpha \mathrm{KG}$ administration. The elevated expression of pAkt in lung of SARS-CoV-2 infected animals was significantly reduced after $\alpha \mathrm{KG}$ supplementation (Figure 6K-L). Besides, an elevated expression of HIF $2 \alpha$ in the lung of SARS-CoV-2 infected animals was significantly reduced after $\alpha$ KG supplementation (Supplemental Figure 13A-B). The body weight decreased significantly in infected animals during day 3 - day 6 , but no rescue effect of $\alpha \mathrm{KG}$ on body weight was observed (Figure 6M). As reported by others (27), we did not observe death of SARS-CoV-2 infected hamsters. We also observed a gradual increase in body weight on day 8 onwards in another group of all infected hamsters. However, a decreased viral load in the lung was observed at day 6 in infected animals supplemented with $\alpha \mathrm{KG}$ (Figure 6N). Supplementation with $1 \%$ dietary $\alpha \mathrm{KG}$ for 6 days to control hamsters did not alter the count of blood cells including platelet and WBCs and granulocytes (Supplemental Figure 8D-F), suggesting a safe implementation of the metabolite.

\section{Discussion}

Our study for the first time describes the regulatory role of PHD2-pAkt axis in platelet function. Also, we show heretofore unreported inactivating impact of $\alpha$-ketoglutarate $(\alpha \mathrm{KG})$ mediated augmentation of prolyl hydroxylation activity of PHD2 on phosphorylated Akt1 (pAkt1). An earlier study has described that pAkt1 undergoes prolyl hydroxylation at Pro125 and Pro313 by PHD2 in a reaction decarboxylating $\alpha \mathrm{KG}$. Hydroxylated pAkt1(Thr308) is dephosphorylated by Von Hippel-Lindau protein (pVHL) associated protein phosphatase 2A (PP2A), leading to Akt inactivation. Study unveils this pathway as another line of post translational modification for pAkt. In VHL-deficient/suppressed setting and under hypoxic microenvironment, accumulation of pAkt is likely to promote tumour growth and its inhibition partially reverses the effect (25).

We describe here that the supplementation with $\alpha \mathrm{KG}$, an intermediate of TCA cycle, significantly suppresses pAkt1 and reduces agonist-induced platelet activation under normoxia. 
Upon activation by agonists like collagen (3) and ADP (30) platelets undergo Akt phosphorylation by PDK1. Akt phosphorylation is known to stimulate cell surface adhesion molecules like GPIIbIIIa and GPVI, and promote platelet aggregation and adhesion, and also secretion of granular contents (31-32). $\alpha \mathrm{KG}$ supplementation significantly inhibits the above -mentioned functions of platelets by suppressing pAkt1. Contrastingly, a marked amplification in platelet activity alongside increased pAkt1 was observed after treatment with PHD2 inhibitors like DKG or DHB. This indicates a crucial involvement of PHD2 in the regulation of platelet activation. The above speculation was further supported by the $\alpha$ KG-mediated reduced expression of HIF $1 \alpha$ and HIF $2 \alpha$, known substrates of PHD2, and DKG/DHB-driven stabilization of the same. Augmented PHD2 activity orchestrated these events was confirmed by increased hydroxylated proline alongside enhanced binding of PHD2 to pAkt in $\alpha \mathrm{KG}$-treated platelets. Besides, our study also describes an increased intracellular $\alpha \mathrm{KG}$ : succinate ratio in platelets after $\alpha \mathrm{KG}$ supplementation. PHD2 catalyses proline hydroxylation of its substrate by converting $\mathrm{O}_{2}$ and $\alpha \mathrm{KG}$ to $\mathrm{CO}_{2}$ and succinate (33), and succinate can inhibit PHD2 by competing with $\alpha \mathrm{KG}$ (34). Therefore, an elevated intracellular ratio of $\alpha \mathrm{KG}$ to succinate may serve as a stimulator of PHD2 activity as suggested (28). It is notable that $\alpha \mathrm{KG}$ : succinate ratio was unaltered in platelet after activation with agonist, but its elevated ratio in $\alpha$ KG-supplemented platelet significantly augmented PHD2 activity and in turn downmodulated pAkt1. Contrastingly, several studies have reported an increased pAkt in platelets and other cells in vitro (35-36) and in vivo (37) after succinate supplementation. Thus, indicating that intracellular ratio of these two metabolites serves as a switch for the PHD2 activity. We show that $\alpha \mathrm{KG}$ supplementation significantly decreased pAkt in platelet to inhibit aggregation, thrombus formation and secretion of granular contents including inflammatory mediator such as sphingosin-1 phosphate (S1P) in vitro. S1P is one of the connecting between platelet activation and systemic inflammation as it can activate monocytes. Interestingly we observed that $\alpha \mathrm{KG}$ could also suppress S1P-mediated activation of monocytes in a pAkt1 dependent manner. When LPS was used as an activator to simulate a thrombo-inflammatory condition, $\alpha \mathrm{KG}$ could deter the secretion of pro-inflammatory cytokines from monocytes. Importantly, our data showed no significant inactivation of pAkt1 by $\alpha \mathrm{KG}$ in PHD2-deficient monocytic cell line, thus confirming that $\alpha \mathrm{KG}$ imparts its effects primary through PHD2 in this context. We could also confirm that the outcomes of $\alpha \mathrm{KG}$ usage were independent of HIF1 $\alpha$. Overall, our data upholds PHD2 as a 
potential target to abrogate Akt signalling. Studies have extensively used antagonists/inhibitors to target pathological signalling of Akt or PI3K-Akt to inhibit thrombosis and inflammation (8, 4, 23-24).

Our study highlights the implementation of dietary $\alpha \mathrm{KG}$ to mice as one of the potential treatments to reduce the platelet aggregation and inflammatory response of monocyte by downmodulating pAkt1 without altering the count of these cell types. Therefore, it suggests a safe administration of this metabolite. In a recent work, we have described a safe rescue effect of this metabolite in mice from hypoxia-induced inflammation by downmodulating HIF $\alpha$ (29). $\alpha \mathrm{KG}$ has been used extensively for in vivo experimental therapies for manipulating multiple cellular processes related to organ development and viability of organisms (38-39), restriction of tumor growth and extending survival (40), and preventing obesity (41).

Another interesting part of our study describes the $\alpha \mathrm{KG}$-mediated rescue of clot formation and leukocyte accumulation alongside a reduction in cytokine secretion by these cells in lung and other organs in mice exposed to a thrombosis-inducing agent like carrageenan. Specifically, our study also reports a significant rescue effect of $\alpha \mathrm{KG}$ on inflamed lung in SARS-CoV-2 infected hamsters. A significant reduction in intravascular clot formation and accumulation of leukocytes including macrophages and neutrophils in alveolar spaces of the lung of infected hamsters suggests a potential rescue effect of $\alpha \mathrm{KG}$. Thus, indicating that $\alpha \mathrm{KG}$ usage can decelerate inflammation induced lung tissue damage reported in severe cases of SARS-CoV-2 infection and may eventually deter development of acute respiratory distress syndrome (42-43). However, this speculation needs further experimental evidences. Besides, $\alpha \mathrm{KG}$ treatment also decreased viral load in the lung of the infected animals alongside diminished expression of pAkt. Although the exact role Akt in replication of SARS-CoV-2 remains to be delineated as reported for other viruses (44). But its noteworthy that a recent study has described an elevated pAkt1(Thr308) in cells infected with SARS-CoV-2 (17). Our in vitro data also show an elevated pAkt1(Thr308) alongside increased IL6 secretion by SARS-CoV-2 infected Huh7 cell line, which was further inhibited by $\alpha \mathrm{KG}$ administration. Thus, suggesting that the augmentation of PHD2 activity by $\alpha \mathrm{KG}$ would be a potential therapeutic strategy to inhibit pAkt-mediated anomalies like inflammation and thrombosis in host and also propagation of SARS-CoV-2. 
Therefore, our data together suggest a novel role of PHD2-pAkt axis in the regulation of platelet and leukocyte functions. Supplementation with $\alpha \mathrm{KG}$ significantly increases the hydroxylase activity of PHD2 and therefore reduces phosphorylation of Akt and in turn supresses thrombotic and inflammatory functions of platelets and leukocytes respectively, depicted in schematic Figure 7. Thus, suggesting a safe implementation of dietary $\alpha \mathrm{KG}$ in prevention of Aktdriven thrombosis and inflammation in various disease conditions including inflamed lung in COVID-19. Study also highlights $\alpha$ KG-PHD2-pAkt axis as a potential target for better pulmonary management in these diseases.

\section{Materials and Methods}

\section{Platelet and monocyte isolation}

Whole blood $(16 \mathrm{ml})$ was collected form healthy individuals in sodium citrate or ACD anticoagulant. Platelets and monocytes were isolated from whole blood and used for in vitro experiments. $16 \mathrm{ml}$ of whole blood was collected from healthy volunteers by venepuncture in vacutainers containing anti-coagulant sodium citrate or acid-citrate dextrose (ACD). Platelet rich plasma (PRP) was separated by centrifugation at $44 \mathrm{~g}$ for $15 \mathrm{~min}$. Sodium citrate containing PRP was used for aggregation and activation studies. PRP in ACD was used for isolating washed platelets for further studies as described in our previous work (45).

Peripheral blood mononuclear cells (PBMCs) were isolated using Ficoll Hypaque (GE Healthcare, Freiburg, Germany) density gradient centrifugation as mentioned in our earlier work (46). PBMCs were washed twice with PBS, pH 7.4 and seeded in cell culture-treated plates (Corning, NY, USA) in RPMI-1640 medium (Sigma Aldrich, USA) supplemented with 10\% (v/v) fetal bovine serum (Gibco Invitrogen, San Diego, CA), $100 \mathrm{U} / \mathrm{mL}$ penicillin and $100 \mu \mathrm{g} / \mathrm{mL}$ streptomycin for $2 \mathrm{hrs}$ at $37^{\circ} \mathrm{C}$ in a humidified atmosphere with $5 \% \mathrm{CO}^{2}$, to allow monocytes to adhere to the plate. After $2 \mathrm{hrs,} \mathrm{supernatant} \mathrm{containing} \mathrm{non-adherent} \mathrm{cells} \mathrm{were} \mathrm{removed} \mathrm{and}$ adhered monocytes were used for further treatments mentioned hereafter.

\section{Platelet activation and aggregation assays}

Human PRP diluted (1:1) in Tyrod's buffer $\mathrm{pH} 7.2$ was used for following assays. Diluted PRP was pre-treated with octyl $\alpha$-ketoglutarate (Sigma Aldrich, USA) or inhibitors to PHD2 such as dimethyl ketoglutarate (DKG, Sigma Aldrich) or ethyl-3-4-dihydroxybenzoic acid (DHB, TCI America, Portland $)$ and incubated with collagen $(10 \mu \mathrm{g} / \mathrm{ml})$ or ADP $(2 \mu \mathrm{M}$, both from the Bio/Data 
Corporation, USA) 10 minutes. Platelets were labelled with P-selectin (PE Cy5), PAC-1 (FITC) and Annexin-V (FITC) antibodies (BD bioscience) for $15 \mathrm{~min}$ at $37^{\circ} \mathrm{C}$ and fixed in $1 \%$ paraformaldehyde. 20,000 events were acquired using flow cytometry (BD FACS Verse). The acquired data was analysed using the Flowjo software (Tree Star, USA), as mentioned (45).

Platelet aggregation was performed using PAP8 aggregometer (Bio/Data Corporation, USA). PRP was pre-treated with $\alpha \mathrm{KG}$ or DKG and incubated with collagen $(20 \mu \mathrm{g} / \mathrm{ml})$ or ADP $(5 \mu \mathrm{M})$ and aggregation percentage was measured.

\section{Platelet thrombus formation assay}

Platelet thrombus formation assay was performed by perfusing whole blood (collected in citrateanticoagulant from healthy individuals) over the petri plate immobilized with collagen. Whole blood was preincubated for 5 min with either $\alpha \mathrm{KG}$ or $\mathrm{DKG}$ or both before perfusion on collagen coated surface. A syringe pump (Harvard Apparatus Inc., USA) was connected to the outlet port that drew blood through the chamber at arterial shear stress of $25 \mathrm{dyne} / \mathrm{cm}^{2}$. The flow chamber was mounted onto a Nikon Eclipse Ti-E inverted stage microscope (Nikon, Japan) equipped with a high-speed digital camera. Movies were recorded at magnification 40X and analysed using NISElements version 4.2 software as mentioned in our previous study (47).

\section{Platelet microparticle measurement}

PRP was pre-treated with/without $\alpha \mathrm{KG}$ and incubated with collagen for $10 \mathrm{~min}$. Platelet-free plasma was obtained by 2 sequential centrifugations: PRP at $1500 \mathrm{~g}$ for $7 \mathrm{~min}$ followed by platelet-poor plasma (PPP) at $1500 \mathrm{~g}$ for $15 \mathrm{~min}$. Platelet-derived microparticles (MPs) were measured using flow cytometry after labelling with anti-CD41 PE antibody as mentioned (45).

\section{Monocyte activation}

Primary monocytes and monocytic cell line were pre-treated with $1 \mathrm{mM}$ octyl $\alpha$-ketoglutarate (Sigma Aldrich, USA) for 2 hrs and 4 hrs respectively followed by replacement with fresh media. Cells were treated with either S1P $(1 \mu \mathrm{M})$ or LPS $(500 \mathrm{ng} / \mathrm{ml})$. Treated cell supernatant was used for assessing cytokines using the cytometric bead array (CBA). Protein lysate prepared from cell pellet was used for western blotting of signalling molecules.

\section{CBA for quantifying cytokines}

Cytokines such as TNF- $\alpha$, IL-1 $\beta$, IL-6 and IL-10 were measured from human primary monocytes culture supernatant or mice plasma of different treatments as mentioned in results using CBA and analysed by FCAP array software (BD Biosciences, San Jose, CA,USA). 


\section{Generation of HIF-1 $\alpha$ depleted monocyte cell line}

HIF-1 $\alpha$ protein expression was depleted in human U937 cell line (ATCC, USA) using shRNA targeting HIF-1 $\alpha$ (TCRN0000010819, Sigma Aldrich, USA) using a liposome mediated delivery (Life Technologies, Thermo Fisher Scientific, USA).

\section{Generation of PHD2 depleted monocyte cell line}

PHD2 was depleted in human U937 cell line using shRNA targeting EGLN1 as mentioned our publication (29).

\section{Dietary $\alpha$ KG supplementation to mice and hamsters}

Male BALB/c mice aged 5-7 weeks or Syrian golden hamsters of 8 weeks were supplemented with $1 \%$ of dietary $\alpha$-ketoglutarate (SRL, Mumbai, India) in drinking water for 24 or 48 hrs (to mice), or for 6 days (to hamsters) as mentioned in schematic Figure 4, 5 and 6. The blood cell counts and other assays were performed.

\section{Mice platelet aggregation}

PRP was collected from control and $\alpha \mathrm{KG}$-treated mice and diluted with PBS (1:1 vol) and processed for centrifugation at $90 \mathrm{~g}$ using brake-free deceleration on a swinging bucket rotor for $10 \mathrm{~min}$. Platelets counts were adjusted to $2.5 \times 10^{8} / \mathrm{ml}$ and platelet aggregation was performed using PAP8 aggregometer. Collagen $(7.5 \mu \mathrm{g} / \mathrm{ml})$ or ADP $(5 \mu \mathrm{M})$ was used as aggregation agonist.

\section{Carrageenan treatment to mice and measuring thrombosis and cellularity scores}

$\mathrm{BALB} / \mathrm{c}$ mice were used to develop carrageenan-induced thrombosis model (26). Mice were injected with $100 \mu \mathrm{l}$ of $10 \mathrm{mg} / \mathrm{ml} \kappa$-carrageenan (Sigma Aldrich, USA) prepared in normal saline in intraperitoneal cavity. $\alpha \mathrm{KG}$ was supplemented via drinking water to these mice. After $48 \mathrm{hrs}$ of carrageenan treatment, length of thrombus covered tail was measured and percentage tail thrombosis was calculated by length of thrombus covered tail/total length of tail $\times 100$.

Thrombosis score was measured in lung and liver of above mice. Lungs and liver samples were fixed in $4 \%$ formalin and paraffin embedded. $2.5 \mu \mathrm{m}$ thick sections were prepared and stained with Haematoxylin and Eosin (H\&E) and Masson's trichrome (MT). Slides were observed under Nikon Eclipse Ti-E inverted stage microscope (Nikon, Japan) and images were acquired at $20 \mathrm{X}$ to observe thrombi and at $40 \mathrm{X}$ to observe leukocyte accumulation. Thrombosis scoring was calculated using ImageJ software. Thrombi were selected using freehand selection tool in MT stained slides. Percentage area covered was calculated as percentage of freehand selected area covering the total area. Similarly, leukocyte accumulation was assessed as a marker of 
inflammation in the above lung section using percentage cellularity. Cellularity score was calculated using ImageJ software. Images were converted to RGB stack and from that all nuclei were selected based on the intensity of color and size from H\&E stained slides. Percentage area covered by nucleated cells was calculated by measuring nuclear area as a percentage of the total tissue section area.

Carrageenan-induced peritoneal inflammation was measured in mice using a modified protocol as described (48). BALB/c mice received carrageenan (10 $\mathrm{mg} / \mathrm{ml})$ or saline intraperitoneally. At $3 \mathrm{hrs}$ and $6 \mathrm{hrs}$, the animals were anaesthetized and peritoneal exudates were harvested in $3 \mathrm{ml}$ of PBS. Different immune cell populations in peritoneal lavage were analysed and counts were determined by flow cytometry using CD45.2, CD11b, CD11c, Ly6G, Ly6C, and CD41 (46, 49). In another set of a similar experiment, BALB/c mice were injected with carrageenan. At 3 and $6 \mathrm{hrs}$, peritoneal inflammation was visualised using bioluminescence based imaging of MPO activity by injecting luminol (i.p. $20 \mathrm{mg} / 100 \mathrm{~g}$ body weight) (Sigma Aldrich) 6 min prior to imaging using an in-vivo imaging system (IVIS; Perkin Elmer, Waltham, MA, USA) as mentioned in our work (46).

\section{SARS-CoV-2 infection to Syrian golden hamsters}

Male golden hamsters of 8 weeks old were given infection of SARS-CoV-2 (isolate USA-WA1/2020 from World Reference Center for Emerging Viruses and Arboviruses, from UTMB, Texas, USA), via nasal route inoculation using $1 \times 10^{6}$ plaque-forming units (PFU) as mentioned (27). $1 \%$ dietary $\alpha \mathrm{KG}$ was administered via drinking water and $400 \mu \mathrm{l}$ of $10 \% \alpha \mathrm{KG}$ was given through oral gavage on day 3 through day 5. During this phase they were symptomatic and were not drinking sufficient (male hamster of 100 gm B wt. drinks normally $5 \mathrm{ml}$ per day) water. The schematic protocol of infection and therapy is mentioned in Figure 6. At day-6, animals were sacrificed and lungs and liver samples were harvested, fixed in 4\% formalin, paraffin embedded and processed for H\&E and MT staining. The thrombosis and inflammation scores were measured as mentioned above. The lung sections were used for immunohistochemistry staining for pAkt (Cell Signalling Tech, USA) Body weight was recorded on alternate days. The lung sections were used for measuring viral genome using RT-PCR. 
Human liver cell line Huh7 (ATCC, USA) was seeded $\left(6 \times 10^{4}\right.$ cells/well) and pre-treated with $1 \mathrm{mM}$ octyl $\alpha \mathrm{KG}$ for $2 \mathrm{hrs}$ and infected with $0.1 \mathrm{MOI}$ of SARS-CoV-2 for $24 \mathrm{hrs}$ in BSL3 facility. The cells pellet was lysed and fixed for using estimation of pAkt1 using western blotting.

\section{RT-PCR detection of viral genome}

Lung tissue sample from hamsters was homogenized in Trizol reagent (MRC, UK) using a handheld tissue-homogenizer and the total RNA extracted as per manufacturer's protocol. $1 \mu \mathrm{g}$ total RNA was reverse-transcribed using Superscript-III reverse-transcriptase (Invitrogen, USA) as per manufacturer's protocol, using random hexamers (Sigma Aldrich, USA). The cDNA was diluted in nuclease-free water (Promega, USA) and used for real-time PCR with either SARS-CoV-2 or GAPDH specific primers, using 2x SYBR-green mix (Takara Bio, Clontech, USA) in an Applied Biosystems $^{\circledR}$ QuantStudio ${ }^{\mathrm{TM}} 6$ Flex Real-Time PCR System. The oligonucleotides used were SARS-F (5'-CAATGGTTTAACAGGCACAGG-3') and SARS-R (5'CTCAAGTGTCTGTGGATCACG-3') for SARS-CoV-2, and G3PDH-F (5'GACATCAAGAAGGTGGTGAAGCA-3') and G3PDH-R (5'CATCAAAGgTGGAAGAGTGGGA-3'). The Ct value corresponding the viral RNA was normalised to that of G3PDH transcript. The relative level of SARS-CoV-2 RNA in mock-infected samples was arbitrarily taken as 1 and that of infected samples expressed as fold-enrichment (FE). The FE value for each infected sample was transformed to their logarithmic value to the base of 10 and plotted.

The viral genome was measured in Huh7 cell line treated with SARS-CoV-2 using RT-PCR as mentioned above. The $\mathrm{Ct}$ value corresponding the viral RNA was normalised to that of RNAse P (RP) transcript. The oligonucleotides used were RP-F (5'-AGATTTGGACCTGCGAGCG-3') and RP-R (5'-GAGCGGCTGTCTCCACAAGT-3').

\section{Immunoprecipitation}

PHD2 was immunoprecipitated from platelet lysate using protein G sepharose beads and antiPHD2 antibody (Cell Signalling Tech, USA). Similarly, phosphorylated Akt (pAkt) was immunoprecipitated using protein A sepharose beads and anti-pAkt antibody (Cell Signalling Tech, USA). Briefly, washed platelets isolated from whole blood of healthy volunteer and lysed in lysis buffer (25 mM Tris, $150 \mathrm{mM}$ sodium chloride, $1 \mathrm{mM}$ EDTA, 1\% NP-40, $50 \mathrm{mM}$ sodium fluoride and 3\% Glycerol) with protease and phosphatase inhibitor. Lysate was pre-cleared with protein A or G sepharose beads and then added to antibody coated beds and incubated overnight. 
Beads were removed, washed with lysis buffer and collected protein sample was processed for western blotting.

\section{Western blotting}

The whole cell (platelets or primary monocyte or monocytic cell line) lysate was prepared using RIPA lysis buffer and protease-phosphatase inhibitor (Thermo Scientific Life Tech, USA). SDSPAGE gel was followed by immunoblotting using primary antibodies against pAkt, Akt, pAkt1(Thr 308), Akt1, HIF-1 $\alpha$, HIF-2 $\alpha$, $\beta$-Actin (Cell Signalling, USA) and $\alpha$-tubulin (Thermo Fisher Scientific, USA) as described in detail in our previous work (45). The detailed information of antibodies is mentioned in Supplemental Table 1.

\section{Estimation of metabolites}

Steady-state level of $\alpha$-ketoglutarate, Lactate, Fumarate, Pyruvate and Succinate was estimated in plasma, PBMC-granulocytes $\left(10^{5}\right)$ and platelets $\left(10^{5}\right)$ of mice or human samples from different treatments as per manufacture protocol (Sigma Aldrich, USA catalog no. MAK054, MAK064, MAK060, MAK071, MAK335 respectively).

\section{Sphingosine-1-Phosphate measurement}

$\alpha \mathrm{KG}$ treated and control mice platelets were stimulated with collagen $(10 \mu \mathrm{g} / \mathrm{ml})$ in vitro and supernatant was collected and used to estimate Sphingosine-1-phosphate (S1P) level as per manufacturer's protocol [Cloud clone corp. (CEG031Ge)]. Similarly, human platelets were treated with $\alpha \mathrm{KG}$ and collagen $(5 \mu \mathrm{g} / \mathrm{ml})$ and supernatant was used to estimate S1P level.

\section{Statistical Analysis}

Data from at least three experiments are presented as Mean \pm SEM (Standard Error of the Mean). Statistical differences among experimental sets were analysed by Unpaired t test. Graph Pad Prism version 8.0 software was used for data analysis and $P$-values $<0.05$ were considered statistically significant.

\section{Supplemental Materials}

Supplemental Figure 1: Measuring: Akt1 phosphorylation (pAkt1) in platelet induced by ADP; agonist induced pAkt1 in platelet in presence of PHD2 inhibitor DHB; and pPI3K inn presence of $\alpha \mathrm{KG}$. 
Supplemental Figure 2: IgG control for immunoprecipitation of PHD2 and P-Akt from platelet lysate.

Supplemental Figure 3: Microparticle release from platelets after $\alpha \mathrm{KG}$ treatment.

Supplemental Figure 4: ADP induced platelet aggregation in presence of $\alpha \mathrm{KG}, \mathrm{DKG}$ and DHB.

Supplemental Figure 5: Effect of $\alpha \mathrm{KG}$ on LPS induced HIF-2 $\alpha$ and P-Akt expression in monocytes.

Supplemental Figure 6: Generation of HIF-1 $\alpha$ depleted cells.

Supplemental Figure 7: Measuring intracellular level of fumarate and pyruvate in activated platelets after $\alpha \mathrm{KG}$ supplementation.

Supplemental Figure 8: Mice and hamster blood counts after dietary $\alpha \mathrm{KG}$ supplementation.

Supplemental Figure 9: In vitro platelet aggregation in presence of octyl $\alpha \mathrm{KG}$, platelets were collected from normal mice; and platelet aggregation collected from $\alpha \mathrm{KG}$ treated mice.

Supplemental Figure 10: H\&E staining of mice lung and liver showing reduction of carrageenan induced thrombosis by dietary $\alpha-K G$ supplementation.

Supplemental Figure 11: Masson's trichrome (MT) staining of mice lung showing reduction of leukocyte infiltration in carrageenan-treated mice after dietary $\alpha \mathrm{KG}$ supplementation.

Supplemental Figure 12: Gating strategy for immune cells from mice peritoneal lavage fluid.

Supplemental Figure 13: HIF-2 $\alpha$ expression in lung tissue of hamsters infected with SARS-CoV2.

Supplemental Figure 14: Densitometry of western blots.

Supplemental Table 1: List of antibodies used in the study.

\section{Study approval}

Human samples: Ethics approval was obtained from the Institutional Ethics Committee (IEC) for human research of Regional Centre for Biotechnology (RCB; ref no. RCB-IEC-H-08) to recruit healthy volunteers. Volunteers were recruited on the basis of inclusion criteria: 1) healthy, 2) not taking any anti-platelet or anti-inflammatory drugs, 3) no major illness or chronic disease, and 4) no microbial infections within a month of recruitment. A written informed consent was received from all participants.

Animal study: Ethics approval was obtained from the Institutional Animal Ethics Committee (IAEC) of RCB (ref. no. RCB/ IAEC/2020/077) and mice experiments were conducted within the 
guidelines of IAEC in the Small Animal Facility (SAF) of our institute. Ethics approval was obtained from the IAEC (ref. no. RCB/IAEC/2020/069) and Institutional Biosafety Committee (IBSC; ref. no. RCB/IBSC/20-21/221) of RCB and hamster experiments were conducted within the guidelines of IAEC in the BSL3 facility of SAF of our institute.

\section{Authors contributions}

NMS designed and performed all experiments, analysed data and wrote the manuscript. SA designed and performed mice experiment for immunophenotyping and histochemistry and analysed related data. SK and Sankar B have designed and performed covid-19 infection studies, and Sankar B has supervised the infection experiments. Sulagna B designed and performed metabolite estimation and analysed data, and edited the manuscript. JTP provided crucial conceptual inputs. PG designed and supervised the study, conceptualized the approach, designed the experiments, analyzed the data, and wrote the manuscript. All authors read, edited and approved the final manuscript.

\section{Acknowledgements}

Authors acknowledge generous help of Prof. Sudhanshu Vrati of Regional Centre for Biotechnology, Faridabad, India for sharing SARS-CoV-2 virus strain. Authors also acknowledge the funding by grants: BT/PR22881 and BT/PR22985 from the Department of Biotechnology (DBT), Govt. of India; and CRG/000092 from the Science and Engineering Research Board, Govt. of India to PG.

\section{Conflict of interest}

The authors have declared that no conflict of interest exists.

\section{References}

1. M. Dittrich, I. Birschmann, S. Mietner, A. Sickmann, U. Walter, T. Dandekar, Platelet protein interactions: Map, signaling components, and phosphorylation groundstate. Arterioscler. Thromb. Vasc. Biol. 28, 1326-1331 (2008).

2. D. Woulfe, H. Jiang, A. Morgans, R. Monks, M. Birnbaum, L. F. Brass, Defects in secretion, aggregation, and thrombus formation in platelets from mice lacking Akt2. J. Clin. Invest. 113, 441-450 (2004).

3. J. Chen, S. De, D. S. Damron, W. S. Chen, N. Hay, T. V. Byzova, Impaired platelet responses to thrombin and collagen in AKT-1-deficient mice. Blood 104, 1703-1710 (2004).

4. H. Yin, A. Stojanovic, N. Hay, X. Du, The role of Akt in the signaling pathway of the glycoprotein Ib-IX-induced platelet activation. Blood 111, 658-665 (2008). 
5. K. A. O’Brien, A. Stojanovic-Terpo, N. Hay, X. Du, An important role for Akt3 in platelet activation and thrombosis. Blood 118, 4215-4223 (2011).

6. A. Stojanovic, J. A. Marjanovic, V. M. Brovkovych,X. Peng, N. Hay, R. A. Skidgel, X. Du, A phosphoinositide 3-kinase-AKT-nitric oxide-cGMP signaling pathway in stimulating platelet secretion and aggregation. J. Biol. Chem. 281, 16333-16339 (2006).

7. K. A. O’Brien, T. K. Gartner, N. Hay, X. Du, ADP-stimulated activation of akt during integrin outside-in signaling promotes platelet spreading by inhibiting glycogen synthase kinase-3 $\beta$. Arterioscler. Thromb. Vasc. Biol. 32, 2232-2240 (2012).

8. J. C. Reséndiz, M. H. Kroll, R. Lassila, Protease-activated receptor-induced Akt activation Regulation and possible function. J. Thromb. Haemost. 5, 2484-2493 (2007).

9. M. Holinstat, A. M. Preininger, S. B. Milne, W. J. Hudson, H. A. Brown, H. E. Hamm, Irreversible platelet activation requires protease-activated receptor 1-mediated signaling to phosphatidylinositol phosphates. Mol. Pharmacol. 76, 301-313 (2009).

10. M. Camps, T. Rückle, H. Ji,V. Ardissone, F. Rintelen, J. Shaw, C. Ferrandi, C. Chabert, C. Gillieron, B. Françon, T. Martin, D. Gretener, D. Perrin, D. Leroy, P. Vitte, E. Hirsch, M. P. Wymann, R. Cirillo, M. K. Schwarz, C. Rommel, Blockade of PI3K $\gamma$ suppresses joint inflammation and damage in mouse models of rheumatoid arthritis. Nat. Med. 11, 936-943 (2005).

11. M. Sospedra, R. Martin, Immunology of Multiple Sclerosis. Semin. Neurol. 36, 115-127 (2016).

12. W. W. Busse, R. F. J. R. Lemansk, Epidemiologic and clinical observations have linked IgE antibodies to the severity of asthma 13 and the ini- tial and sustained responses of the airway to allergens. 14 To initiate the synthesis of IgE, inhaled allergens must. English J. 344, 350362 (2001).

13. S. D. Shapiro, COPD unwound. N. Engl. J. Med. 352, 2016-9 (2005).

14. M. P. Schön, W. H. Boehncke, Medical Progress: Psorasis. N. Engl. J. Med. 352, 1899-912 (2005).

15. C. Fernández-Hernando, E. Ackah, J. Yu,Y. Suarez, T. Murata, Y. Iwakiri, J. Prendergast, R. Q. Miao, M. J. Birnbaum, W. C. Sessa, Loss of Akt1 Leads to Severe Atherosclerosis and Occlusive Coronary Artery Disease. Cell Metab. 6, 446-457 (2007).

16. J. Paez, W. R. Sellers, PI3K/PTEN/AKT pathway. A critical mediator of oncogenic signaling. Cancer. Treat. Res. 115, 145-67 (2003).

17. R. M. Hekman, A. J. Hume, R. K. Goel, K. M. Abo, J. Huang, B. C. Blum, R. B. Werder, E. L. Suder, I. Paul, S. Phanse, A. Youssef, K. D. Alysandratos, D. Padhorny, S. Ojha, A. MoraMartin, D. Kretov, P. E. A. Ash, M. Verma, J. Zhao, J. J. Patten, C. Villacorta-Martin, D. Bolzan, C. Perea-Resa, E. Bullitt, A. Hinds, A. Tilston-Lunel, X. Varelas, S. Farhangmehr, U. Braunschweig, J. H. Kwan, M. McComb, A. Basu, M. Saeed, V. Perissi, E. J. Burks, M. D. Layne, J. H. Connor, R. Davey, J. X. Cheng, B. L. Wolozin, B. J. Blencowe, S. Wuchty, S. M. Lyons, D. Kozakov, D. Cifuentes, M. Blower, D. N. Kotton, A. A. Wilson, E. Mühlberger, A. Emili, Actionable Cytopathogenic Host Responses of Human Alveolar Type 2 Cells to SARSCoV-2. Mol. Cell. 80, 1104-1122 (2020).

18. M. Bouhaddou, D. Memon, B. Meyer, K. M. White, V. V. Rezelj, M. Correa Marrero, B. J. Polacco, J. E. Melnyk, S. Ulferts, R. M. Kaake, J. Batra, A. L. Richards, E. Stevenson, D. E. Gordon, A. Rojc, K. Obernier, J. M. Fabius, M. Soucheray, L. Miorin, E. Moreno, C. Koh, Q. D. Tran, A. Hardy, R. Robinot, T. Vallet, B. E. Nilsson-Payant, C. Hernandez-Armenta, A. Dunham, S. Weigang, J. Knerr, M. Modak, D. Quintero, Y. Zhou, A. Dugourd, A. Valdeolivas, 
T. Patil, Q. Li, R. Hüttenhain, M. Cakir, M. Muralidharan, M. Kim, G. Jang, B. Tutuncuoglu, J. Hiatt, J. Z. Guo, J. Xu, S. Bouhaddou, C. J. P. Mathy, A. Gaulton, E. J. Manners, E. Félix, Y. Shi, M. Goff, J. K. Lim, T. McBride, M. C. O'Neal, Y. Cai, J. C. J. Chang, D. J. Broadhurst, S. Klippsten, E. De Wit, A. R. Leach, T. Kortemme, B. Shoichet, M. Ott, J. Saez-Rodriguez, B. R. tenOever, R. D. Mullins, E. R. Fischer, G. Kochs, R. Grosse, A. García-Sastre, M. Vignuzzi, J. R. Johnson, K. M. Shokat, D. L. Swaney, P. Beltrao, N. J. Krogan, The Global Phosphorylation Landscape of SARS-CoV-2 Infection. Cell 182, 685-712 (2020).

19. T. Mizutani, S. Fukushi, M. Saijo, I. Kurane, S. Morikawa, Importance of Akt signaling pathway for apoptosis in SARS-CoV-infected Vero E6 cells. Virology 327, 169-74 (2004).

20. C. J. Lee, C. L. Liao, Y. L. Lin, Flavivirus activates phosphatidylinositol 3-kinase signaling to block caspase-dependent apoptotic cell death at the early stage of virus infection. J. Virol. 79, 8388-8399 (2005).

21. M. Hanada, J. Feng, B. A. Hemmings, Structure, regulation and function of PKB/AKT - A major therapeutic target. Biochim Biophys Acta-Proteins. Proteomics. 16973-16 (2004).

22. A. Di Lorenzo, C. Fernández-Hernando, G. Cirino, W. C. Sessa, Akt1 is critical for acute inflammation and histamine-mediated vascular leakage. Proc. Natl. Acad. Sci. U. S. A. 106, 14552-14557 (2009).

23. R. A. Pengal, L. P. Ganesan, G. Wei, H. Fang, M. C. Ostrowski, S. Tridandapani, Lipopolysaccharide-induced production of interleukin-10 is promoted by the serine/threonine kinase Akt. Mol. Immunol. 43, 1557-1564 (2006).

24. D. Nandy, R. Janardhanan, D. Mukhopadhyay, A. Basu, Effect of hyperglycemia on human monocyte activation. J. Investig. Med. 59, 661-667 (2011).

25. J. Guo, A. A. Chakraborty, P. Liu,W. Gan, X. Zheng, H. Inuzuka, B. Wang, J. Zhang, L. Zhang, M. Yuan, J. Novak, J. Q. Cheng, A. Toker, S. Signoretti, Q. Zhang, J. M. Asara, W. G. Kaelin $\mathrm{Jr}$, W. Wei, pVHL suppresses kinase activity of Akt in a proline- hydroxylationdependentmanner. Science 353, 929-932 (2017).

26. M. Hagimori, S. Kamiya, Y. Yamaguchi, M. Arakawa, Improving frequency of thrombosis by altering blood flow in the carrageenan-induced rat tail thrombosis model. Pharmacol. Res. 60, 320-323 (2009).

27. S. F. Sia, L. M. Yan, A. W. H. Chin,K. Fung, K. T. Choy, A. Y. L. Wong, P. Kaewpreedee, R. A. P. M. Perera, L. L. M. Poon, J. M. Nicholls, M. Peiris, H. L. Yen,Pathogenesis and transmission of SARS-CoV-2 in golden hamsters. Nature 583, 834-838 (2020).

28. E. D. MacKenzie, M. A. Selak, D. A. Tennant, L. J. Payne, S. Crosby, C. M. Frederiksen, D. G. Watson, E. Gottlieb, Cell-Permeating $\alpha$-Ketoglutarate Derivatives Alleviate Pseudohypoxia in Succinate Dehydrogenase-Deficient Cells. Mol. Cell. Biol. 27, 3282-3289 (2007).

29. S. Bhattacharya, N. M. Shrimali, G. Mohammad, P. A, Koul, J. T. Prchal, P. Guchhait, Gain-of-function Tibetan PHD2D4E;C127S variant suppresses monocyte function: A lesson in inflammatory response to inspired hypoxia. EBioMedicine 2021;68:103418.

30. S. Kim, C. Foster, A. Lecchi,T. M. Quinton, D. M. Prosser, J. Jin J, M. Cattaneo, S. P. Kunapuli, Protease-activated receptors 1 and 4 do not stimulate Gi signaling pathways in the absence of secreted ADP and cause human platelet aggregation independently of Gi signaling. Blood 99, 3629-3636 (2002).

31. S. J. Shattil, P. J. Newman, W. Dc, Integrins : dynamic scaffolds for adhesion and signaling in platelets Integrins : dynamic scaffolds for adhesion and signaling in platelets. Blood 104, 16061615 (2004). 
32. D. S. Woulfe, Akt signaling in platelets and thrombosis. Expert. Rev. Hematol.3, 81-91 (2010).

33. W. G. Kaelin, P. J. Ratcliffe, Oxygen Sensing by Metazoans: The Central Role of the HIF Hydroxylase Pathway. Mol. Cell. 30, 393-402 (2008).

34. K. L. Gorres, R. T. Raines, Prolyl 4-Hydroxylase. Crit. Rev. Biochem. Mol. Biol. 45, 106-24 (2010).

35. C. Hogberg, O. Gidlof, C.Tan, S. Svensson, J. Nilsson-Ohman, D. Erlinge, B. Olde. Succinate independently stimulates full platelet activation via cAMP and phosphoinositide 3-kinase-b signaling. J Thromb Haemost, 9: 361-72 (2011).

36. T. Wang, Ya-Q. Xu, Ye-X Yuan, P-W. Xu, C. Zhag, F. Li, Li-Na Wang, C Yin, L Zhang, XC. Cai, C-J. Zhu, J-R. Xu, B-Q. Liang, S. Schaul, P-P. Xie, D. Yue, Z-R. Liao, L-L. Yu, L. Luo, G. Zhau, J-P. Yang, Z-H. He, M. Du, Y-P Zhou, B-C Deng, S-B. Wang, P. Gao, X-T. Zhu, Q-Y. Xi, Y-L. Zhang, G. Shu, Q-Y. Jiang. Succinate induces skeletal muscle fiber remodeling via SUCNR1 signaling, EMBO Reprort, 20:e47892 (2019).

37. L. Yang, D. Yu, R. Mo, J. Zhang, H. Hua, L. Hu, Y. Feng, S. Wang, W-y. Zhang, N. Yin, XM. Mo. The Succinate Receptor GPR91 Is Involved in Pressure Overload-Induced Ventricular Hypertrophy. PLoS ONE 11(1): e0147597 (2016).

38. R..M. Chin, X. Fu, M. Y. Pai,L. Vergnes, H. Hwang, G. Deng, S. Diep, B. Lomenick, V. S. Meli, G. C. Monsalve, E. Hu, S. A. Whelan, J. X. Wang, G. Jung, G. M. Solis, F. Fazlollahi, C. Kaweeteerawat, A. Quach, M. Nili, A. S. Krall, H. A. Godwin, H. R. Chang, K. F. Faull, F. Guo, M. Jiang, S. A. Trauger, A. Saghatelian, D. Braas, H. R. Christofk, C. F. Clarke, M. A. Teitell, M. Petrascheck, K. Reue, M. E. Jung, A. R. Frand, J. Huang, The metabolite $\alpha-$ ketoglutarate extends lifespan by inhibiting ATP synthase and TOR. Nature 510, 397-401 (2014).

39. Y. Wang, P. Deng, Y. Liu, Y. Wu, Y. Chen, Y. Guo, S. Zhang, X. Zheng, L. Zhou, W. Liu, Q. Li, W. Lin, X. Qi, G. Ou, C. Wang, Q. Yuan Alpha-ketoglutarate ameliorates age-related osteoporosis via regulating histone methylations. Nat. Commun. 11, 1-14 (2020).

40. T. Q. Tran, E. A. Hanse, A. N. Habowski,H. Li, M. B. I. Gabra, Y. Yang, X. H. Lowman, A. M. Ooi, S. Y. Liao, R. A. Edwards, M. L. Waterman, M. Kong, $\alpha$-Ketoglutarate attenuates Wnt signaling and drives differentiation in colorectal cancer. Nat. Cancer. 1, 345-358 (2020).

41. Q. Tian, J. Zhao, Q. Yang, B. Wang, J. M. Deavila, M. J. Zhu, M. Du, Dietary alphaketoglutarate promotes beige adipogenesis and prevents obesity in middle-aged mice. Aging Cell 19, 1-10 (2020).

42. M. Ackermann, S. E. Verladen, M. Kuehnel, A. Haverich, T. Welte, F. Laenger, A. Vanstapel, C. Werlein, H. Stark H, A. Tzankov, W. W. Li, V. W. Li, S. J. Mentzer, D. Jonigk, Pulmonary vascular endothelialitis, thrombosis and angiogenesis in COVID-19. N. Engl. J. Med. 383, 120128 (2020).

43. P. D. McMullen, J. H. Cho, J. L. Miller, A. N. Husain, P. Pytel, T. Krausz, A Descriptive and Quantitative Immunohistochemical Study Demonstrating a Spectrum of Platelet Recruitment Patterns Across Pulmonary Infections Including COVID-19. Am. J. Clin. Pathol. 155, 354-363 (2021).

44. L. Galluzzi, C. Brenner, E. Morselli, Z. Touat, G. Kroemer. Viral control of mitochondrial apoptosis. PLoS Pathogen, 4, e1000018 (2008)

45. R. Singhal, G. K. Annarapu, A. Pandey,S. Chawla, A. Ojha, A. Gupta, M. A. Cruz, T. Seth, P. Guchhait, Hemoglobin interaction with GP1bo induces platelet activation and apoptosis: A novel mechanism associated with intravascular Hemolysis. Haematologica100, 1526-1533 (2015). 
46. A. Bhasym, G. K. Annarapu, S. Saha, N. Shrimali, S. Gupta, T. Seth, P. Guchhait, Neutrophils develop rapid proinflammatory response after engulfing $\mathrm{Hb}$-activated platelets under intravascular hemolysis. Clin. Exp. Immunol. 197, 131-140 (2019).

47. G. K. Annarapu, R. Singhal, A. Gupta,S. Chawla, H. Batra, T. Seth, P. Guchhait, HbS Binding to GP1ba Activates platelets in sickle cell disease. PLoS One 11, 1-12 (2016).

48. M. Torres-Rêgo, A. A. Furtado, M. A. O. Bitencourt,M. C. Lima, R. C. Andrade, E. P. Azevedo, C. Soares Tda, J. C. Tomaz, N. P. Lopes, A. A. da Silva-Júnior, S. M. Zucolotto, F. Fernandes-Pedrosa Mde, Anti-inflammatory activity of aqueous extract and bioactive compounds identified from the fruits of Hancornia speciosa Gomes (Apocynaceae). B. M. C. Complement. Altern. Med. 16, 1-10 (2016).

49. E. E. Ghosn, A. A. Cassado, G. R. Govoni,T. Fukuhara, Y. Yang, D. M. Monack, K. R. Bortoluci, S. R. Almeida, L. A. Herzenberg, L. A. Herzenberg. Two physically, functionally, and developmentally distinct peritoneal macrophage subsets. Proc. Natl. Acad. Sci. U. S. A. 107, 2568-2573 (2010). 
bioRxiv preprint doi: https://doi.org/10.1101/2021.06.11.448037; this version posted August 7, 2021. The copyright holder for this preprint (which was not certified by peer review) is the author/funder, who has granted bioRxiv a license to display the preprint in perpetuity. It is made available under aCC-BY-NC-ND 4.0 International license.

Figure 1

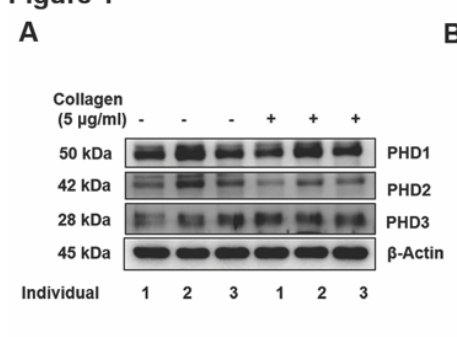

E

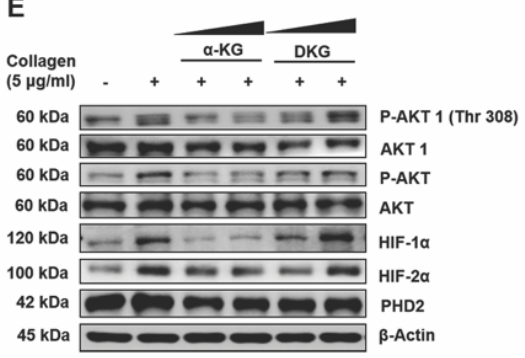

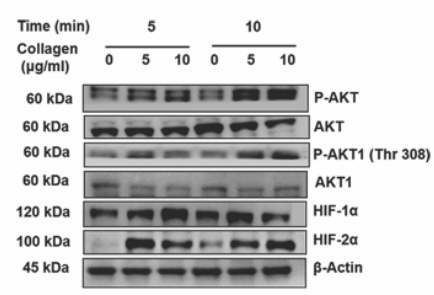

$\mathbf{F}$

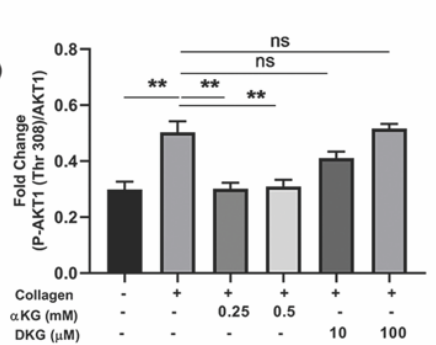

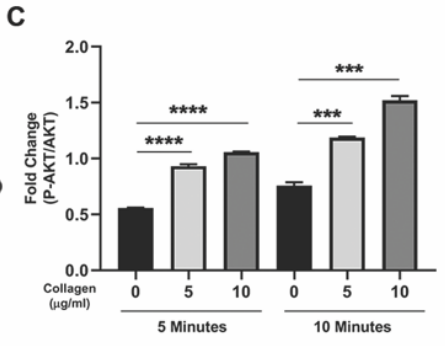

G

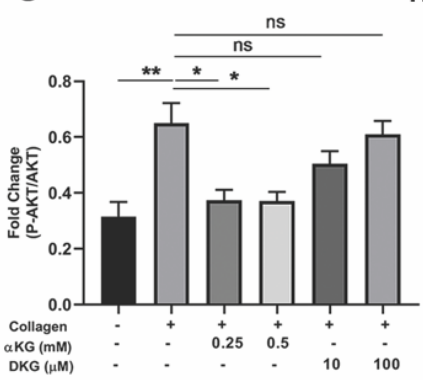

D

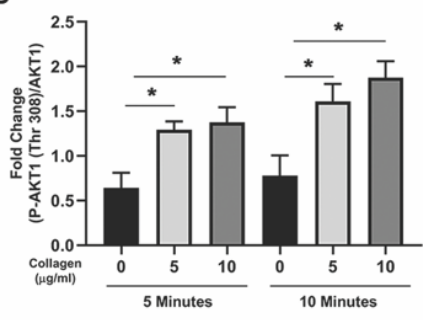

H

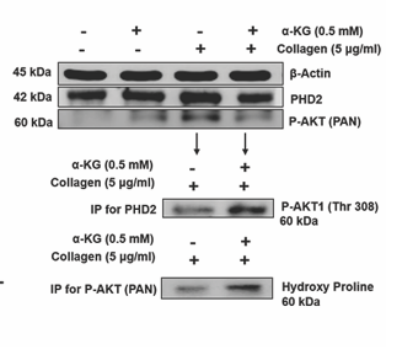

I

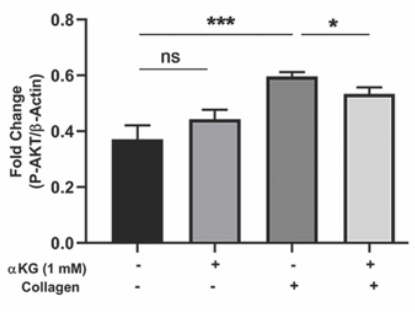

K

L

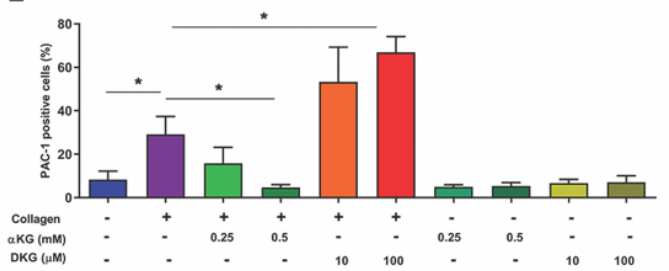

M

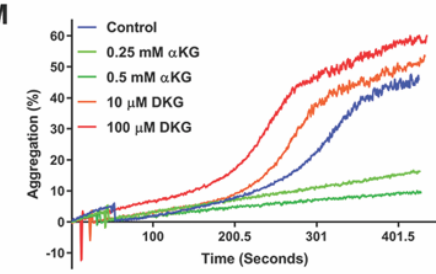

N

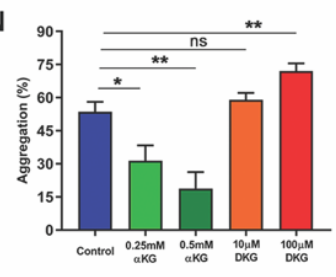

0

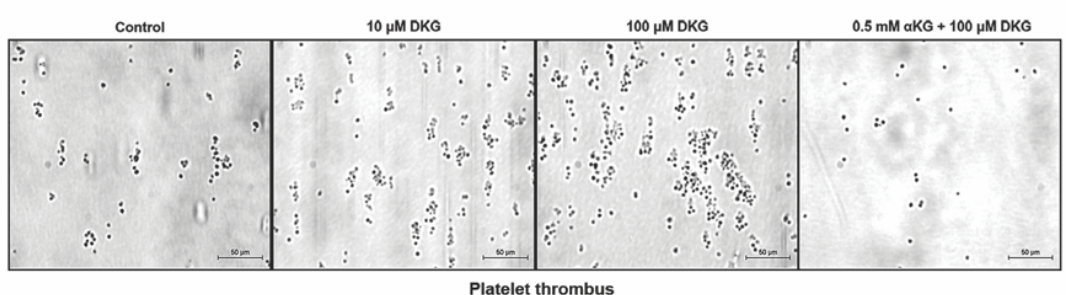

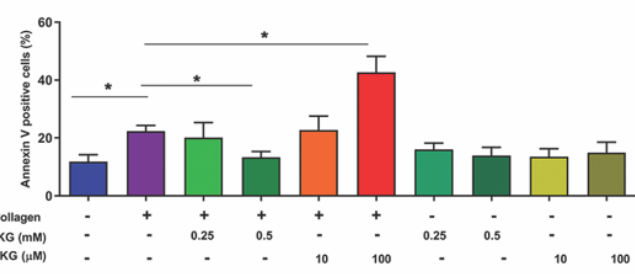

Q

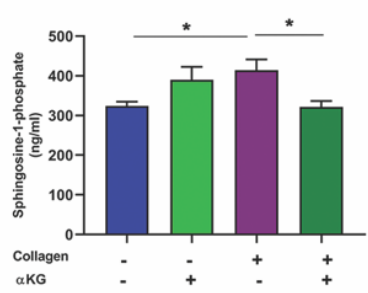


Figure 1. $\alpha$ KG mediated augmentation of prolyl-hydroxylase activity of PHD2 inactivate pAkt in platelets. (A) All 3 isoforms of PHD exist in human platelet. Platelet-rich plasma (PRP) isolated from 3 different healthy individuals, after adjusting to equal number of platelets, was incubated with or without collagen $(5 \mu \mathrm{g} / \mathrm{ml})$ for $5 \mathrm{~min}$ and processed for western blotting (WB) of PHD1, PHD2 and PHD3. Densitometry data are mentioned in Supplemental Figure 14A. (BG) PRP was incubated with collagen in a time- and concentration-dependent manner, and (B) expression of phosphorylated (p)-Akt, Akt, pAkt1(Thr 308), Akt1, HIF-1 $\alpha$ and HIF-2 $\alpha$ was measured in platelet pellet using WB. (C-D) Densitometry analysis shows elevated expression of pAkt and pAkt1 after collagen stimulation, other densitometry data are mentioned in Supplemental Figure 14B-C. (E) Platelets were incubated with collagen in presence of $\alpha \mathrm{KG}(0.25$ and $0.5 \mathrm{mM})$ or DKG $(10$ and $100 \mu \mathrm{M})$ and the expression of above signaling molecules and PHD2 was measured using WB. (F-G) Densitometry data show suppression of collagen-induced elevation of pAkt and pAktl by $\alpha \mathrm{KG}$, but an elevation of these molecules in presence of DKG. Other densitometry data are mentioned in Supplemental Figure 14D-E. (H-I) (H) Immunoprecipitation (IP) of PHD2 from lysate of washed-platelets from above experiment was performed and processed for WB of pAkt; further, IP of pAkt from same lysate and WB for hydroxy proline shows the interaction between the molecules. (I) Densitometry data of pAkt. Other densitometry data are mentioned in Supplemental Figure 14F-G. (J-L) PRP from above experiment of Figure $1 \mathrm{C}$ was processed for measuring surface $(\mathrm{J})$ P-Selectin, (K) PS (Annexin-V binding) and (L) GPIIbIIIa activation (PAC-1 binding) using flow cytometry. (M-N) (M) Platelet aggregation was performed using PRP pre-treated with $\alpha \mathrm{KG}$ or DKG in response to collagen. (N) Percentage platelet aggregation was measured. Data from similar experiment in response to agonist ADP are mentioned in Supplemental Figure 4. (O-P) (O) PRP from healthy individuals was incubated with $\alpha \mathrm{KG}$ or $\mathrm{DKG}$ and perfused on immobilized collagen surface under arterial flow share condition $25 \mathrm{dyne} / \mathrm{cm}^{2}$ and platelet thrombus formation was measured. Scale bar $50 \mu \mathrm{m}$. (P) Thrombus area was measured. (Q) Secretion of Sphingosine-1-phosphate (S1P) was quantified from supernatant of $\alpha \mathrm{KG}$ - and collagen-treated washed platelets using ELISA. Data in above figure are Mean \pm SEM from 3 independent experiments. Unpaired t-test was used to compare between the groups, $* P<0.05 . * * P<0.01, * * * P<0.001, * * * * P<0.0001$ and ns=non-significant. 
bioRxiv preprint doi: https://doi.org/10.1101/2021.06.11.448037; this version posted August 7, 2021. The copyright holder for this preprint (which was not certified by peer review) is the author/funder, who has granted bioRxiv a license to display the preprint in perpetuity. It is made available under aCC-BY-NC-ND 4.0 International license.

Figure 2

A



D

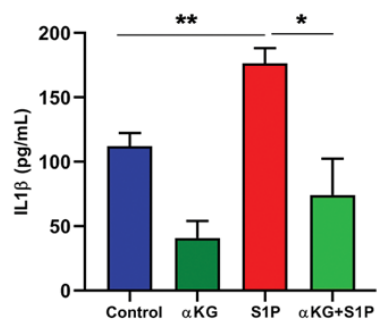

$\mathrm{H}$

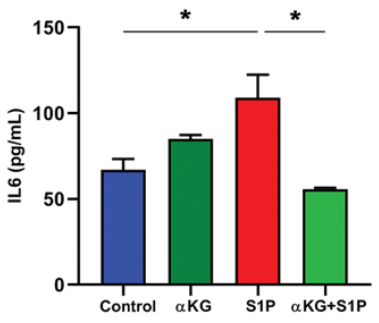

L

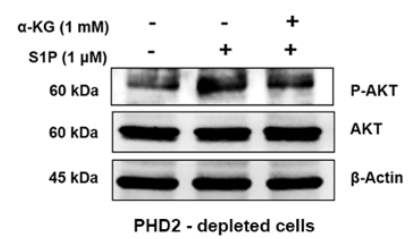

E
B

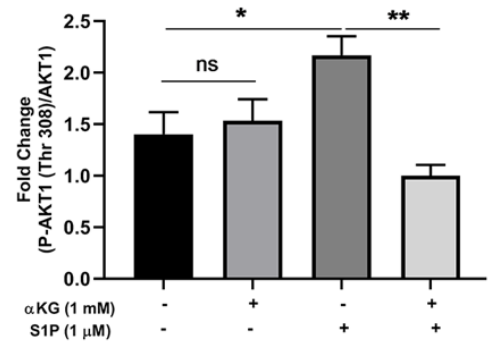

$\mathbf{F}$

C
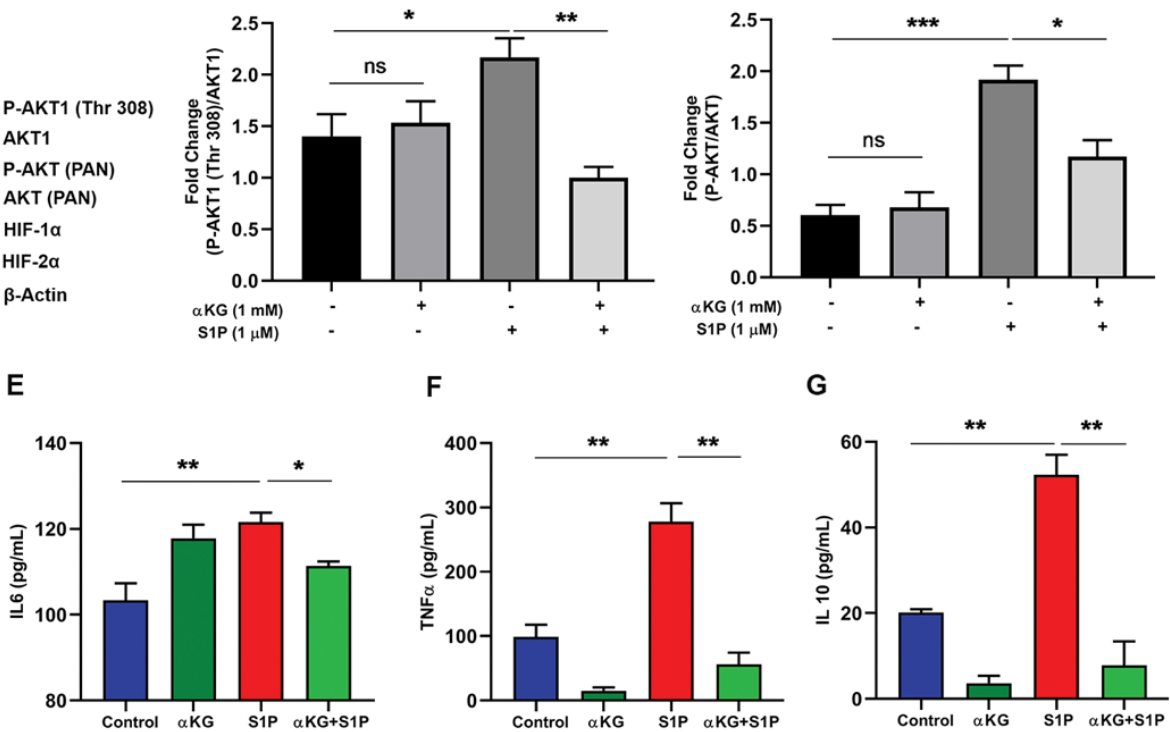

G

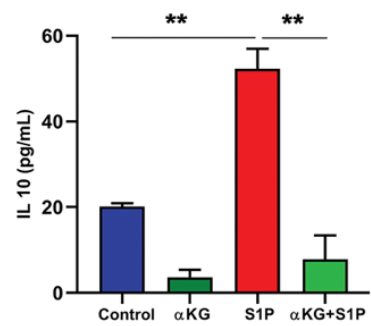

J

K
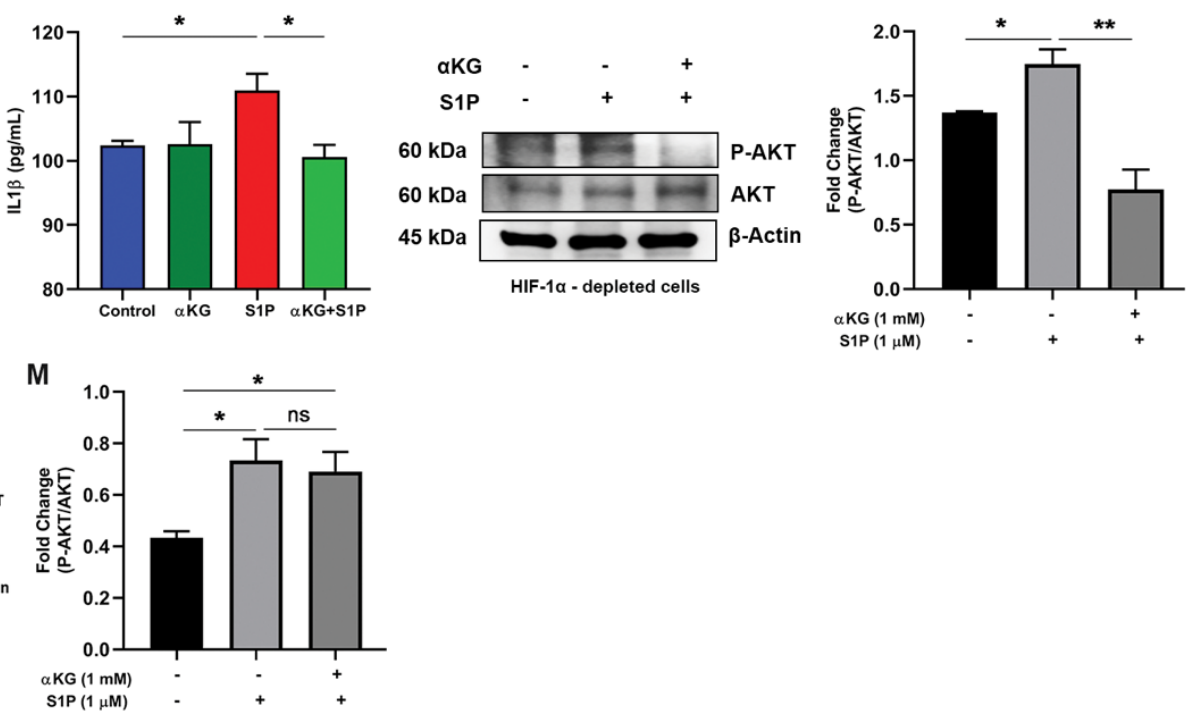
Figure 2. $\alpha$ KG mediated elevation in prolyl-hydroxylase activity of PHD2 inhibits Akt phosphorylation in monocytes. (A-C) Monocytes isolated from healthy individuals were activated with S1P with/without pre-treatment with $\alpha \mathrm{KG}$. (A) The expression of pAkt, Akt, pAkt1(Thr 308), Akt1, HIF-1 $\alpha$ and HIF2 $\alpha$ was measured using WB. (B-C) Densitometry data show suppression of S1P-induced elevation of pAkt and pAkt1 by $\alpha \mathrm{KG}$. Other densitometry data are mentioned in Supplemental Figure 14I-J. Data from similar experiment using LPS as an agonist to monocytes, are mentioned in Supplemental Figure 5. (D-G) The cytokines such as (D) IL1 $\beta$, (E) IL6, (F) TNF $\alpha$ and (G) IL10, secreted by monocytes from above experiment, were measured using flow cytometry based CBA array. (H-K) U937 monocytic cell line was depleted for HIFA (HIF1 $\alpha$ ) using shRNA (detailed protocol is mentioned in Supplemental Figure 6) and treated with $\alpha \mathrm{KG}$ before exposing to S1P treatment. (H) IL6 and (I) IL1 $\beta$ were measured from supernatant. (J$\mathrm{K})$ The cells from above experiment were used for measuring expression of pAkt and Akt using WB. (L-M) PHD2-depleted U937 monocytic cells were treated with $\alpha \mathrm{KG}$ before exposing to S1P treatment and used for measuring expression of pAkt and Akt using WB. Data in above figure are Mean \pm SEM from 3 independent experiments. Unpaired t-test was used to compare between the groups, ${ }^{*} P<0.05, * * P<0.01$ and $\mathrm{ns}=$ non-significant. 
Figure 3

A

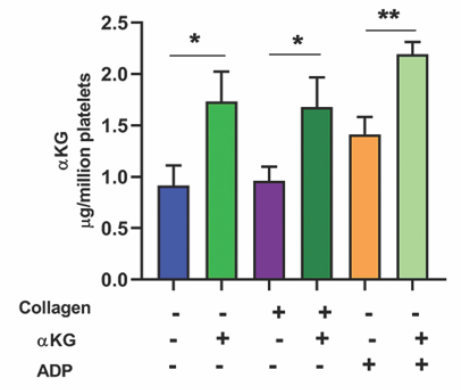

E

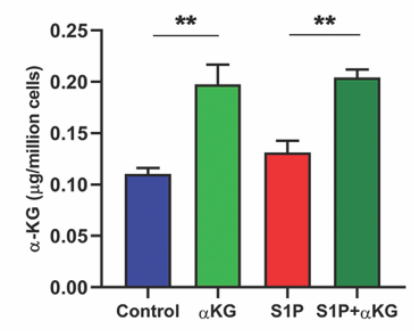

B

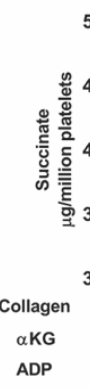

$\mathbf{F}$

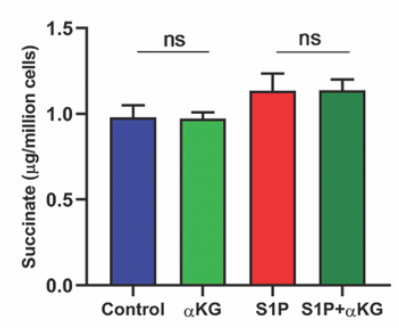

C

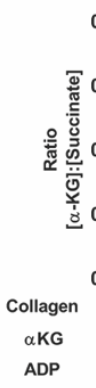

D

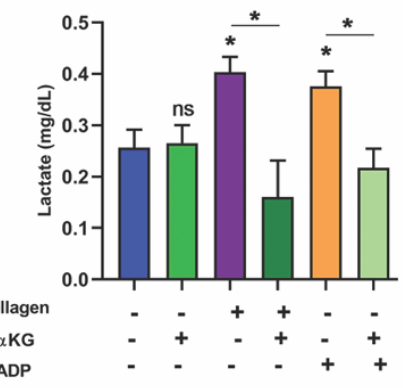

G

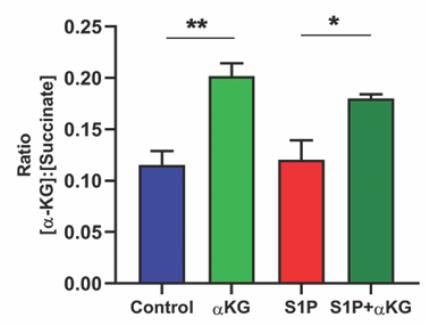

Figure 3. Elevation in intracellular ratio of $\alpha \mathrm{KG}$ to succinate promotes PHD2 activity in both platelet and monocyte. (A-D) PRP isolated from healthy individuals was pre-treated with $\alpha \mathrm{KG}$ and activated in presence of collagen/ADP. Platelet pellet was washed and lysed, and used for measuring intracellular (A) $\alpha \mathrm{KG}$ and (B) succinate using colorimetry based assay. (C) Represents intracellular ratio of $\alpha \mathrm{KG}$ to succinate. (D) Release of Lactate from supernatant of platelets from above experiment was quantified. Data of Pyruvate and Fumarate from platelet lysate is mentioned in Supplemental Figure 7. (E-F) Similarly, intracellular (E) $\alpha \mathrm{KG}$ and (F) succinate were measured from cell lysate of primary monocytes pretreated with $\alpha \mathrm{KG}$ and stimulated with S1P as mentioned in Figure 2A. (G) Represents ratio of $\alpha \mathrm{KG}$ to succinate in monocytes. Data in above figure are Mean \pm SEM from 3 independent experiments in duplicates. Unpaired t-test was used to compare between the groups, ${ }^{*} P<0.05$ and ${ }^{* *} P<0.01$, ns $=$ non-significant. 
bioRxiv preprint doi: https://doi.org/10.1101/2021.06.11.448037; this version posted August 7, 2021. The copyright holder for this preprint (which was not certified by peer review) is the author/funder, who has granted bioRxiv a license to display the preprint in perpetuity. It is made available under aCC-BY-NC-ND 4.0 International license.

Figure 4

A

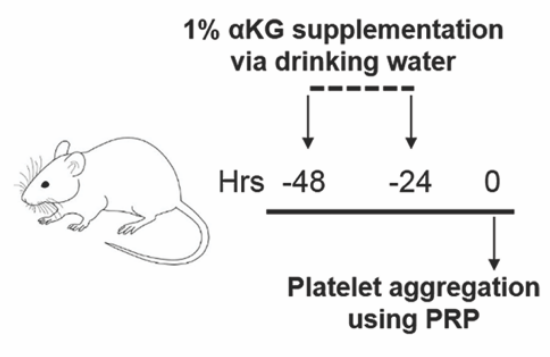

D



H

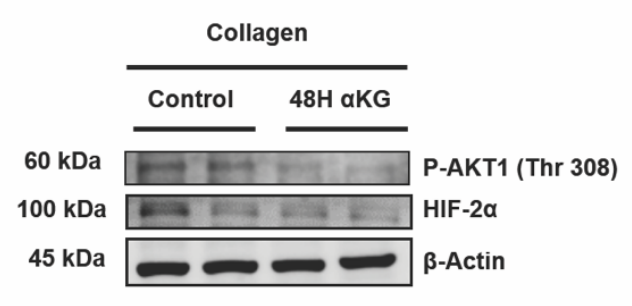

$\mathbf{K}$

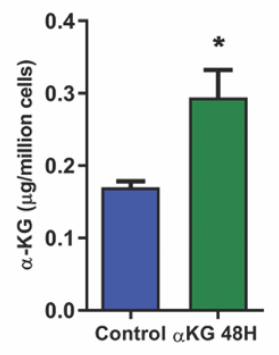

B

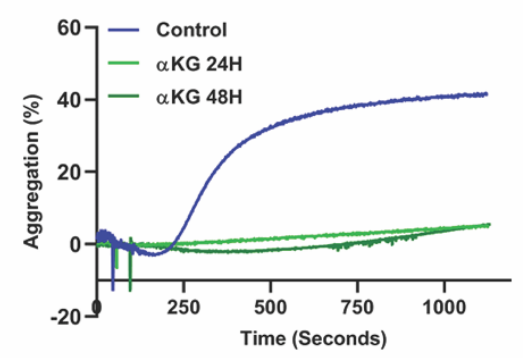

$\mathbf{F}$

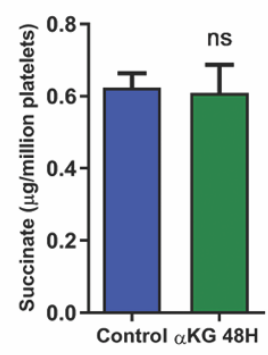

C

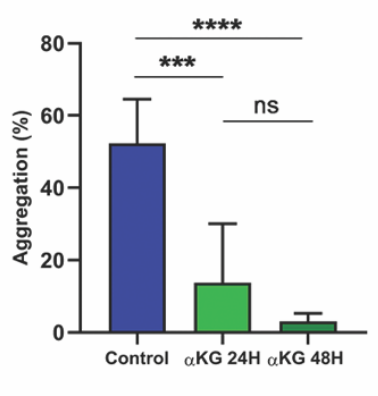

G

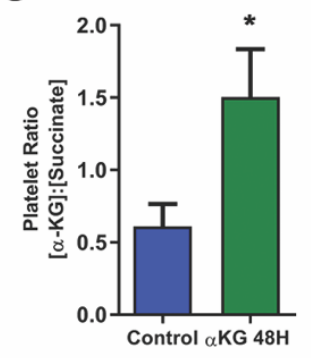

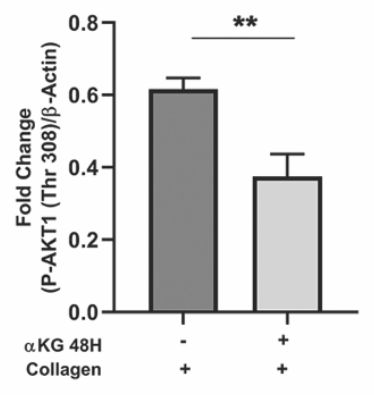

J

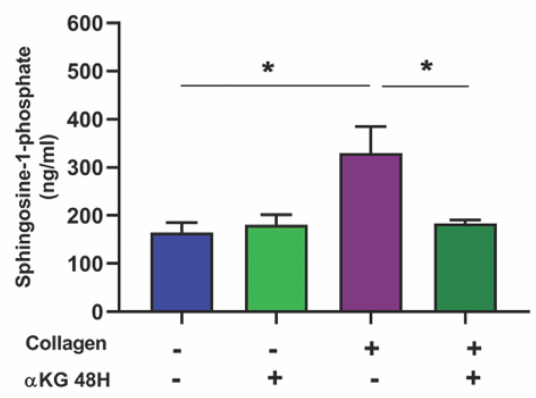

N

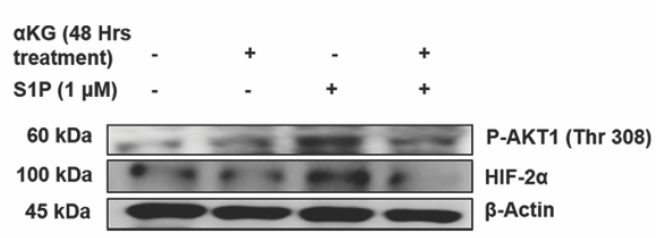

L



M

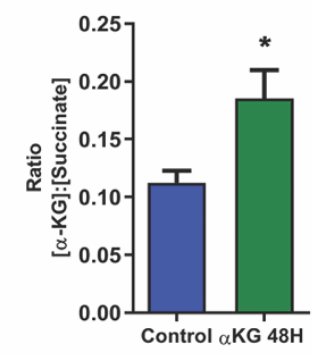

0

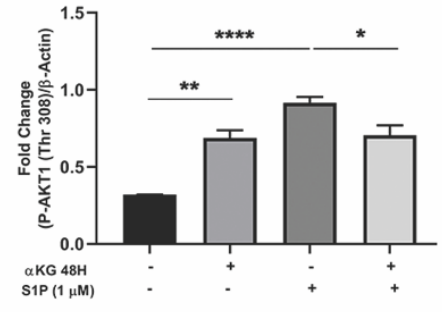


Figure 4. Dietary $\alpha$ KG supplementation to healthy wild type mice reduces platelet and monocyte activation. (A) Schematic showing experimental protocol. BALB/c mice were supplemented with dietary $\alpha \mathrm{KG}$ for 24 and $48 \mathrm{hrs}$, and whole blood was isolated to separate PRP for assessing following parameters. (B-C) (B) Percentage platelet aggregation in presence of collagen or ADP (ADP data in Supplemental Figure 9). (C) Percentage platelet aggregation from 8 mice in each group. (D-G) (D) Plasma concentration of $\alpha \mathrm{KG}$ was quantified from above mice, control vs. $48 \mathrm{hrs} \alpha \mathrm{KG}$ groups $(\mathrm{n}=8)$. The intracellular $(\mathrm{E}) \alpha \mathrm{KG}$ and $(\mathrm{F})$ succinate and their $(\mathrm{G})$ fold change ratio in platelets of mice pre-treated with/without $\alpha \mathrm{KG}$ for 48 hours $(\mathrm{n}=8)$. (H-I) $(\mathrm{H})$ Expression of pAkt1(Thr 308), HIF-2 $\alpha$ was measured in platelets from mice of above groups using WB. Representative image shows blot from 2 mice. (I) Densitometry data show suppression of collagen-induced elevation of pAkt1(Thr 308) in $\alpha \mathrm{KG}$ treated mice $(\mathrm{n}=8)$. Other densitometry data in Supplemental Figure 14K. (J) S1P secretion was measure from supernatant of collagenactivated platelets of above mice groups $(n=8)$. (K-M) Similarly, the intracellular $(K) \alpha K G$ and (L) succinate and their $(\mathrm{M})$ fold change ratio in PBMCs was detected from above mice $(\mathrm{n}=8)$. (NO) (N) Expression of pAkt1(Thr 308), HIF-2 $\alpha$ was measured in PBMCs from above mice, after treating the cells with S1P ex vivo. Representative image shows blot from a mouse. (O) Densitometry data show suppression of S1P-induced elevation of pAkt in $\alpha \mathrm{KG}$ treated mice $(\mathrm{n}=8)$. Other densitometry data in Supplemental Figure 14L. Data in above figure are Mean $\pm \mathrm{SEM}$ from 3 independent experiments. Unpaired t-test was used to compare between the groups, ${ }^{*} P<0.05$, $* * P<0.01, * * * P<0.001, * * * * P<0.0001$ and ns=non-significant. 
bioRxiv preprint doi: https://doi.org/10.1101/2021.06.11.448037; this version posted August 7, 2021. The copyright holder for this preprint (which was not certified by peer review) is the author/funder, who has granted bioRxiv a license to display the preprint in perpetuity. It is made available under aCC-BY-NC-ND 4.0 International license.

Figure 5

A

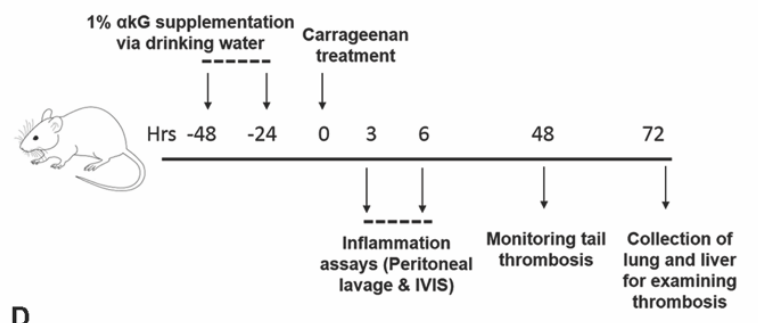

D
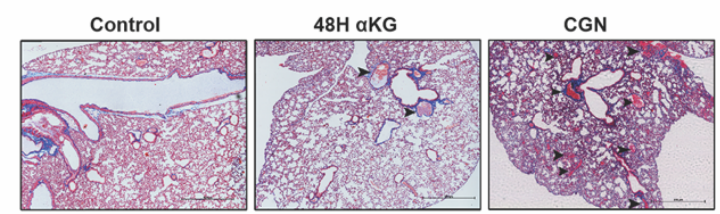

$\mathbf{F}$

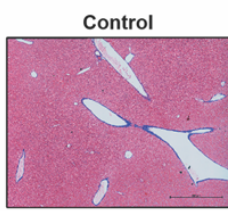

$48 \mathrm{H}$ aKG
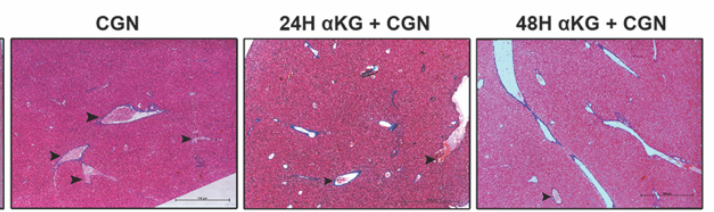

H
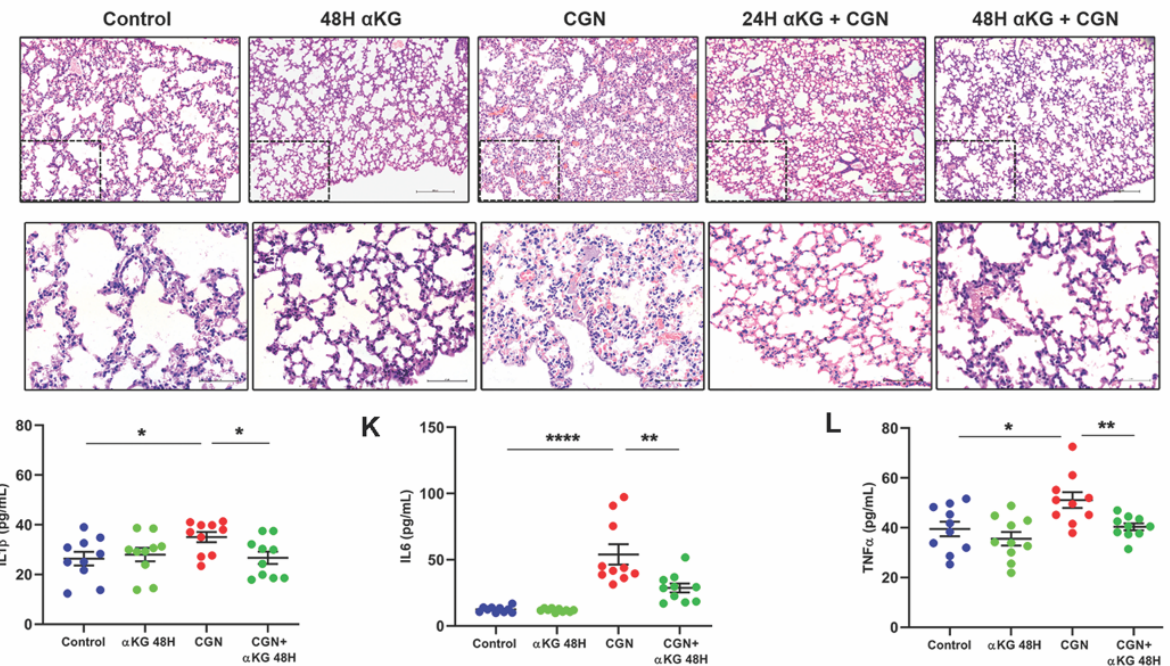

N
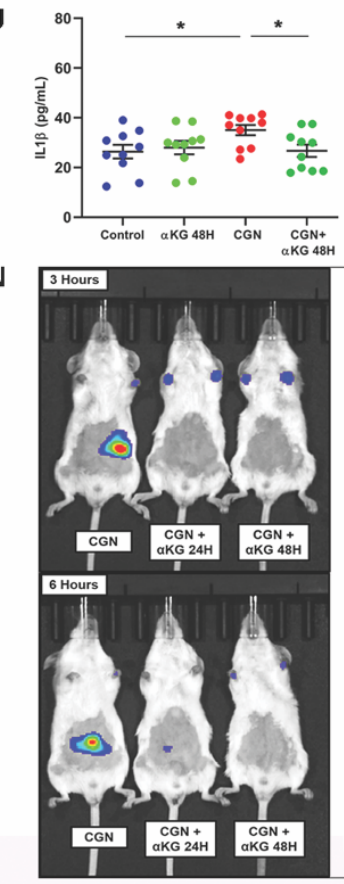

B
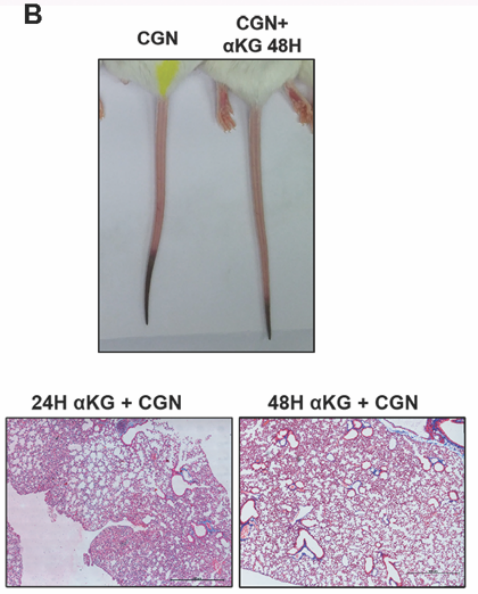

-28.2.

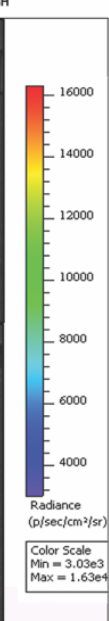

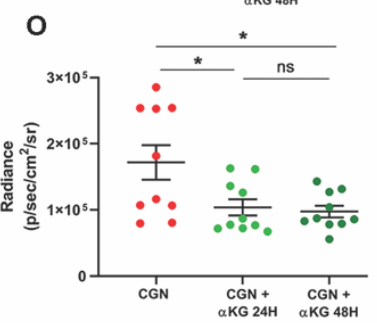

$\mathbf{R}$

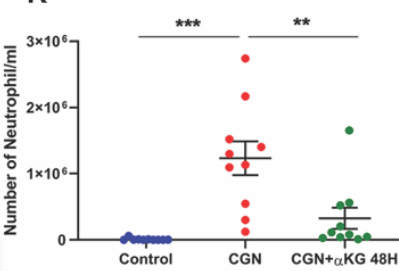

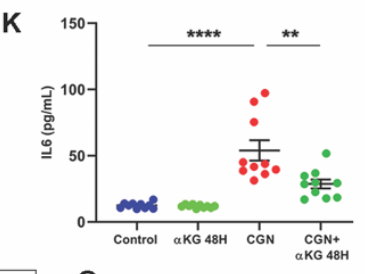

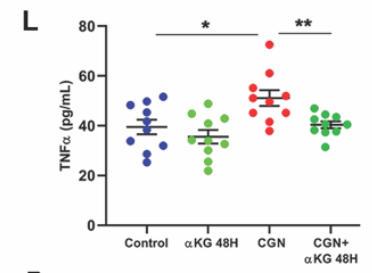

P

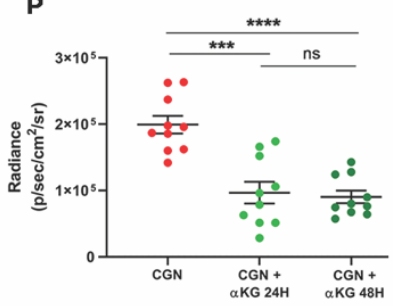

S

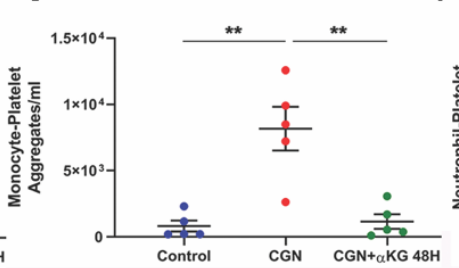

M

Q

T
C

E

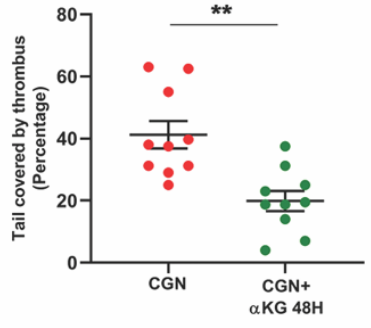

G

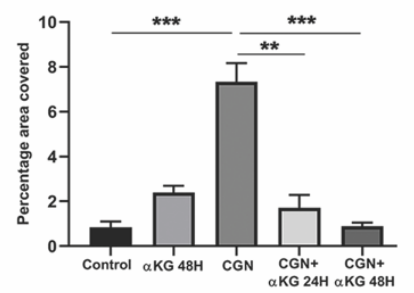

I
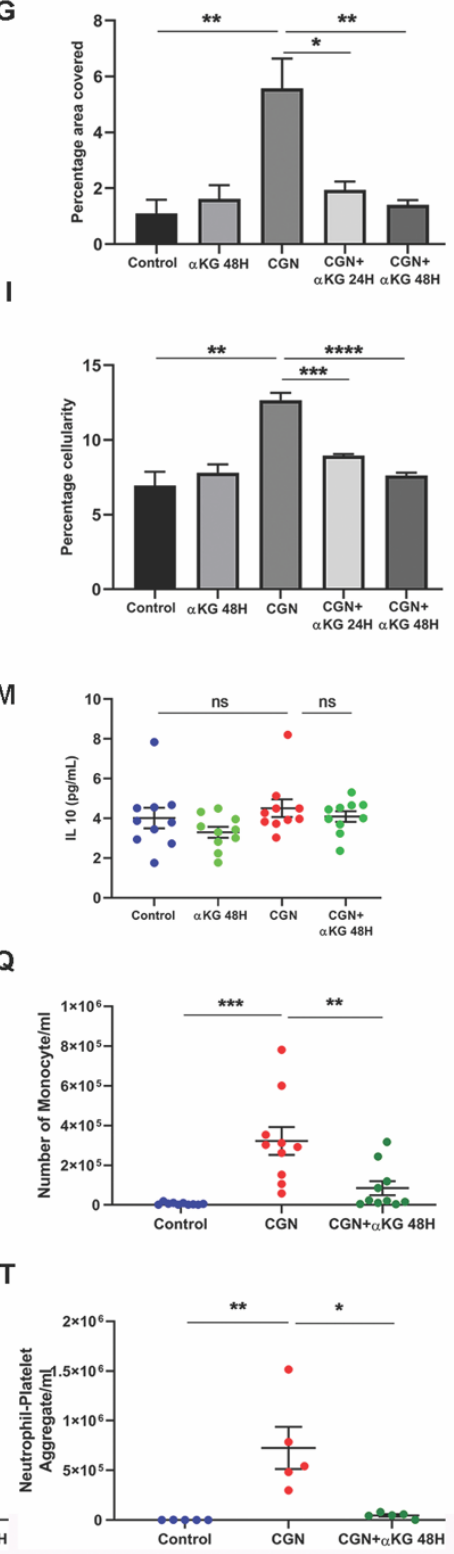
Figure 5. Dietary $\alpha K G$ supplementation rescues mice from carrageenan-induced thrombosis and inflammation. (A) Schematic showing experimental protocol. BALB/c mice were supplemented with dietary $\alpha \mathrm{KG}$ before exposed to carrageenan treatment and following parameters were assessed. (B-C) (B) Tail thrombosis was measured, showing a representative image. (C) Percentage thrombosis. Each dot represents a mouse. (D-G) (D-E) Lung and (F-G) Liver section from above mice groups were used for assessing clot formation using Massontrichrome (MT) staining. Scale bar $500 \mu \mathrm{m}$. Percentage covered area of clot was measured. The hematoxylin and eosin (H\&E) staining data of similar observations are mentioned in Supplemental Figure 10. (H-I) Similarly, H\&E staining of lung was used for assessing leukocyte accumulation. Score is calculated as percentage cellularity. Scale bar $500 \mu \mathrm{m}$ and $20 \mu \mathrm{m}$. Showing reduced inflammation in $\alpha \mathrm{KG}$-treated mice. The MT staining data of similar observations are mentioned in Supplemental Figure 11. Figure D-I, n=8. (J-M) Cytokines (J) IL1 $\beta$, (K) IL6, (L) TNF $\alpha$ and (M) IL10 were measured from mice groups. (N-T) To measure local inflammation $\alpha$ KGsupplemented mice were injected with carrageenan at peritoneum region and $(\mathrm{N})$ luminescence generated from MPO-luminol interaction were measured using IVIS imaging system to assess leukocyte activation and inflammation. Radiance count from (O) 3 and (P) 6 hours post carrageenan treatment. $\alpha \mathrm{KG}$-supplemented mice displayed reduced MPO signal intensity. (Q) Monocytes, (R) Neutrophils, (S) monocytes-platelet and (T) neutrophil-platelet aggregates were measured from peritoneum lavage of above treated mice. Each dot represents a mouse. Data in above figure are Mean \pm SEM from different experiments. Unpaired t-test was used to compare between the groups, $* P<0.05, * * P<0.01, * * * P<0.001, * * * * P<0.0001$ and ns=non-significant. 
bioRxiv preprint doi: https://doi.org/10.1101/2021.06.11.448037; this version posted August 7, 2021. The copyright holder for this preprint (which was not certified by peer review) is the author/funder, who has granted bioRxiv a license to display the preprint in perpetuity. It is made available under aCC-BY-NC-ND 4.0 International license.

Figure 6

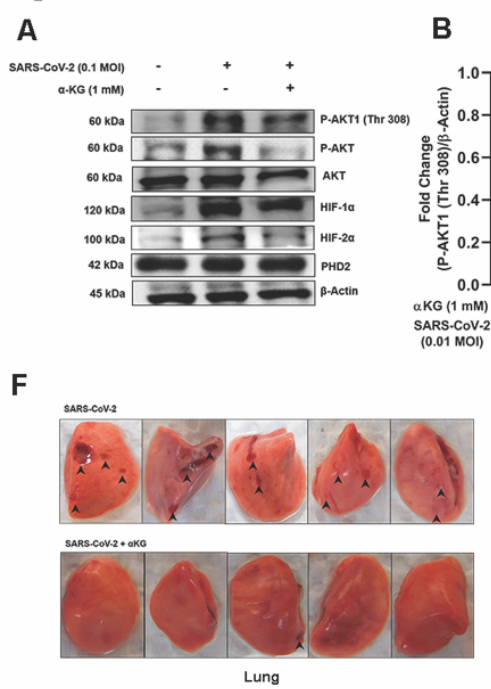

I

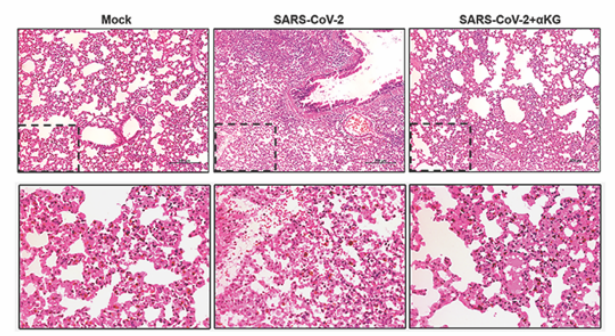

G

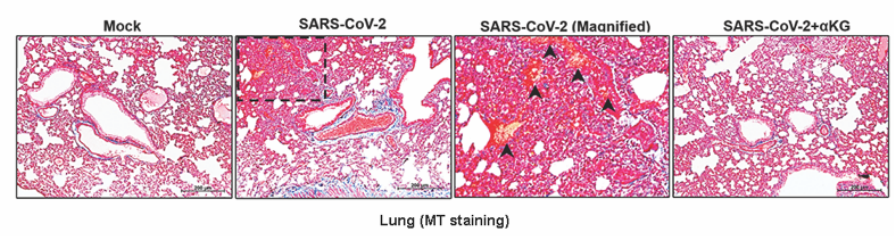

$\mathbf{J}$
C

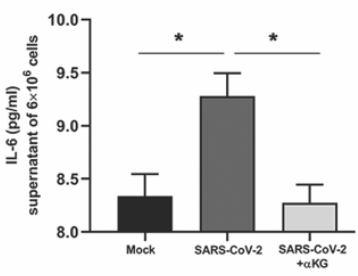

D

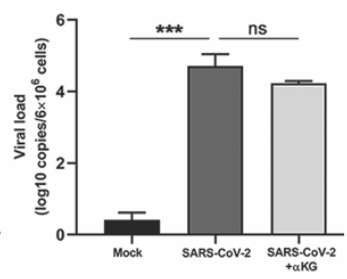

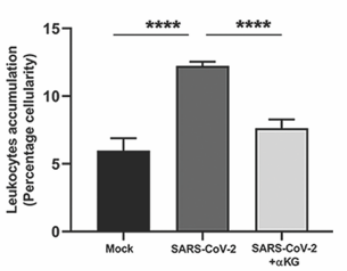

K
E

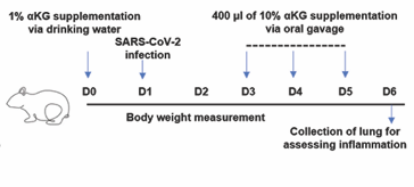

H

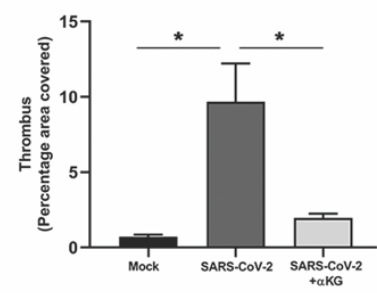

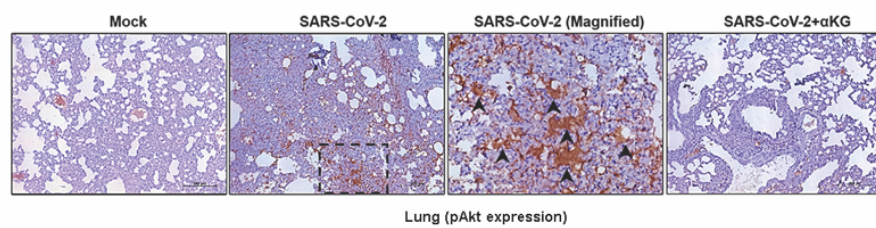

L

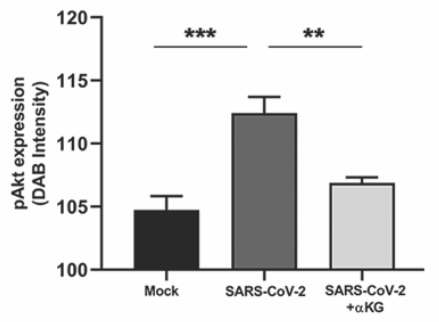

M

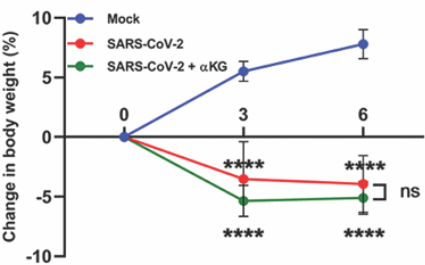

$\mathbf{N}$

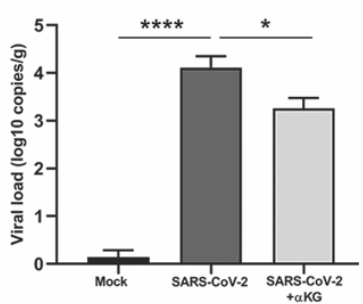


Figure 6. Dietary $\alpha$ KG supplementation rescues hamsters from SARS-CoV-2 induced lung thrombosis and inflammation. (A-B) Before performing in vivo study we performed in vitro experiment and infected human liver Huh7 cell line with 0.1 MOI of SARS-CoV-2 for 24 hrs with a prior treatment with $1 \mathrm{mM}$ octyl- $\alpha \mathrm{KG}$. (A) We assessed pAkt1(Thr308) expression using WB. (B) Densitometry analysis from three independent experiments shows elevated expression of pAkt1(Thr308), other densitometry data are mentioned in Supplemental Figure 14M-P. (C) IL6 level was measured from supernatant of SARS-CoV-2 infected Huh7 cells from above experiment. (D) SARS-CoV-2 genome was measured from above cell pellet using RT-PCR. (E) Schematic Figure shows the experimental protocol using hamsters. (F) Lung was isolated and imaged, showing clot injury spot (arrows) after viral infection, which was rescued to almost normal condition with $\alpha \mathrm{KG}$ supplementation. (G-H) (G) Lung section from above mice groups were used for assessing clot formation and collagen deposition using MT staining and (H) Percentage covered area of thrombus was measured. (I-J) (I) H\&E staining of lung was used for assessing leukocyte accumulation in lung including alveolar spaces as the marker of inflammation. (Lower panel) magnified version from upper panel images. (J) Score is calculated as percentage cellularity. Showing reduced inflammation in $\alpha$ KG-treated animals. (K-L) (K) The sections were labelled with pAkt antibody and stained with HRP-conjugated secondary antibody. (L) pAkt expression was quantified by measuring DAB staining (brown), which was increased in virus infection and decreased after $\alpha \mathrm{KG}$ treatment. (G-L) Image scale bar $200 \mu \mathrm{m}$ and number of animals used $\mathrm{n}=5 \mathrm{in}$ mock, $n=9$ each in other groups. (M) Body weight of the animals were measured at day 0, 3 and 6 , and calculated as Mean $\pm \mathrm{SD}, \mathrm{n}=5$ in mock, $\mathrm{n}=11$ each in other groups. (N) SARS-CoV-2 genome was measured from lung tissue (gram) of hamsters using RT-PCR, $n=3$ in mock, $n=11$ each in other groups. Data in above figure are Mean \pm SEM from independent experiments. Unpaired t-test was used to compare between the groups, $* P<0.05, * * P<0.01, * * * P<0.001$, $* * * * P<0.0001$ and $\mathrm{ns}=$ non-significant. 


\section{Figure 7}

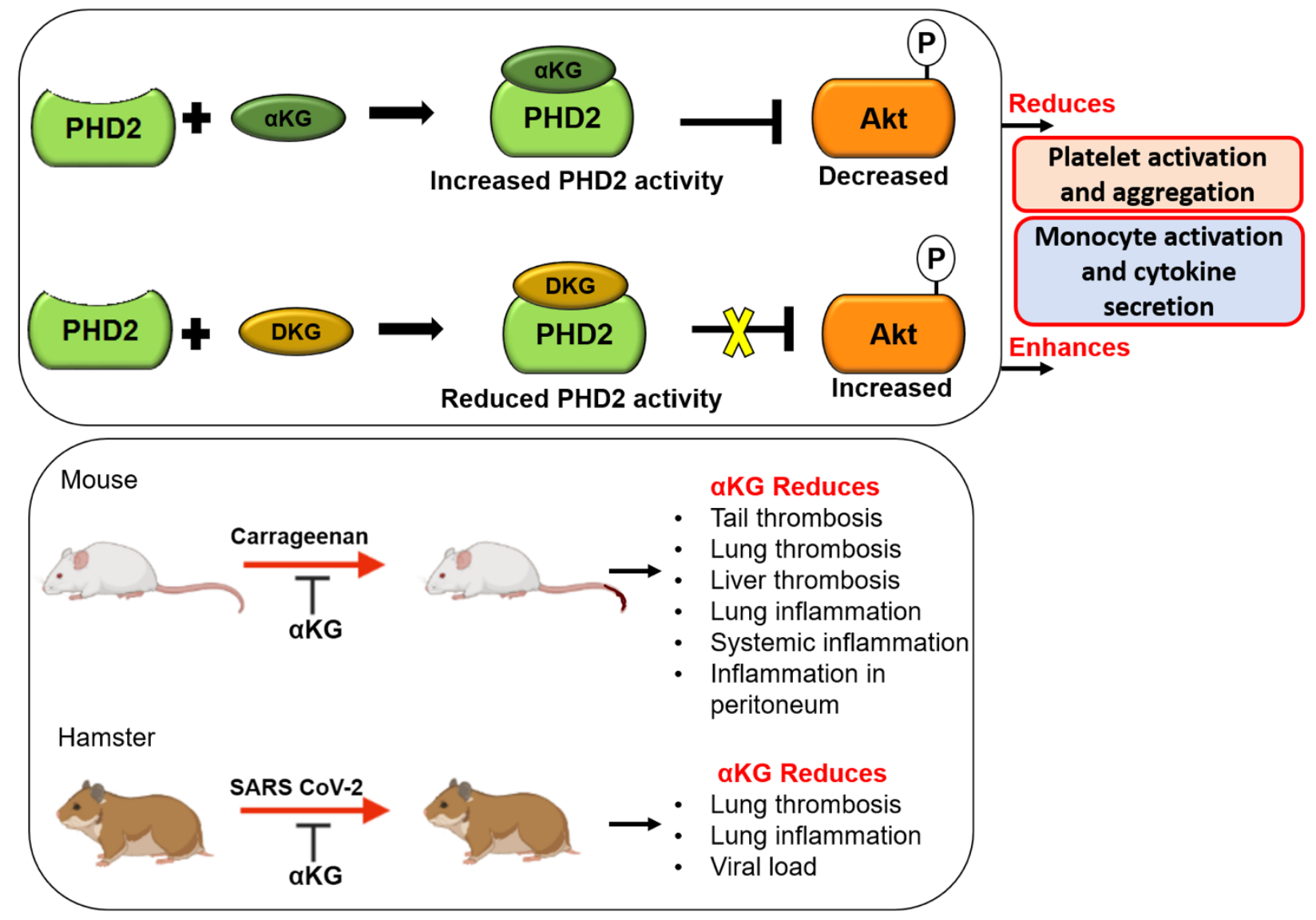

Figure 7. aKG mediated suppression of platelet and monocyte functions. Schematic depicts that under normoxic environment supplementation with $\alpha \mathrm{KG}$ (known cofactor of PHD2) increases PHD2 activity by elevating intracellular $\alpha$ KG: succinate ratio. Elevated PHD2 activity degrades pAkt1 and reduces platelet activation and aggregation. A similar mechanism also suppresses inflammatory function of monocyte. Thus, suggesting the involvement of $\alpha$ KG-PHD2-Akt1 axis in the regulation of events like thrombosis and inflammation. Importantly, dietary $\alpha \mathrm{KG}$ supplementation significantly rescues mice from carrageenan-induced clot formation and leukocyte accumulation and inflammation in various organs including lung. Importantly, dietary $\alpha \mathrm{KG}$ rescues significantly hamsters from SARS-CoV-2 induced clot formation and leukocyte accumulation in the lung, alongside downmodulation of pAkt. 